\title{
Stephanorhinus etruscus (RHINOCEROTIDAE) DEL PLIOCENO SUPERIOR DE CRESPIÀ (GERONA, NE DE ESPAÑA)
}

\author{
David GARCÍA-FERNÁNDEZ', Esperanza CERDEÑO², \\ Àngel GALOBART $T^{3}$ y Xavier ROS \\ ' Museo Geológico del Seminario de Barcelona. C/ Diputación, 231. 08007 Barcelona. \\ España. \\ 2 IANIGLA-CRICYT. Avda. Ruiz Leal, s/n. C.C. 330. 5500 Mendoza, Argentina. \\ ${ }^{3}$ Institut de Paleontologia M. Crusafont. C/ Escola Industrial, 23. 08201 Sabadell, España. \\ + Geoterna. C/ Fray Luis de León, 146A. 08203 Sabadell, España.
}

García-Fernández, D., Cerdeño, E., Galobart, A. y Ros, X. 2001. Stephanorhinus etruscus (Rhinocerotidae) del Plioceno superior de Crespià (Gerona, NE de España). [Stephanorhinus etruscus (Rhinocerotidae) from the later Pliocene of Crespià (Gerona, NE Spain).] Revista Española de Paleontología, 16(1), 145-160. ISSN 0213-6937.

\begin{abstract}
A rather complete rhinoceros skeleton from the late Pliocene (Villafranquian, MN 16-17) of Crespià (BanyolesBesalú Basin, Gerona, Spain) is described in detail. Its preliminary determination as Stephanorhinus etruscus Falconer is confirmed. Other sites where S. etruscus is present are considered for comparisons, as well as some early middle Pleistocene sites with S. hundsheimensis Toula. The skeleton corresponds to an adult individual, with an estimated body mass of $1200 \mathrm{~kg}$. Morphometrically, it shows clear similarities with respect to other Spanish remains of $S$. etruscus such as those from La Puebla de Valverde or El Rincón-1. With respect to other European remains, dimensions are generally below the average values established for this species and the lower premolar series is relatively long. As characteristic morphological details, it can be detached the shortened mandibular symphysis and the relatively low vertical rami of the mandible with respect to other contemporary remains, the upper dentition with less developed crochets than the early Pleistocene remains, and the postcranial bones more slender than those from La Puebla de Valverde, although metapodials have relatively wide epiphyses.
\end{abstract}

Keywords: Stephanorhinus etruscus, Rhinocerotidae, late Pliocene, Villafranchian, Crespià, Spain.

\section{RESUMEN}

Se describe en detalle un esqueleto bastante completo de rinoceronte hallado en el yacimiento plioceno superior (Villafranquiense, MN 16-17) de Crespià (Cuenca de Banyoles-Besalú, Gerona, España). Se confirma su atribución preliminar a Stephanorhinus etruscus Falconer. Se establecen comparaciones con yacimientos comprendidos en el área de distribución bioestratigráfica de la especie y con algunos situados en los niveles inferiores del Pleistoceno medio donde aparece S. hundsheimensis Toula. El esqueleto corresponde a un individuo adulto, con una masa corporal estimada en $1200 \mathrm{~kg}$. A nivel morfométrico, presenta claras semejanzas con otros restos españoles de la especie, como los de La Puebla de Valverde o El Rincón-1. Comparado con otros restos europeos, las dimensiones en general están por debajo de la media establecida para la especie y la serie premolar inferior es relativamente larga. Como detalles morfológicos característicos, destaca la sínfisis mandibular acortada y las ramas ascendentes relativamente bajas con respecto a algunos ejemplares coetáneos; dentición superior con gancho menos desarrollado que los ejemplares del Pleistoceno inferior; y huesos largos más gráciles que los de La Puebla de Valverde, aunque los metápodos mantienen epífisis relativamente anchas.

Palabras clave: Stephanorhinus etruscus, Rhinocerotidae, Plioceno superior, Villafranquiense, Crespià, España. 


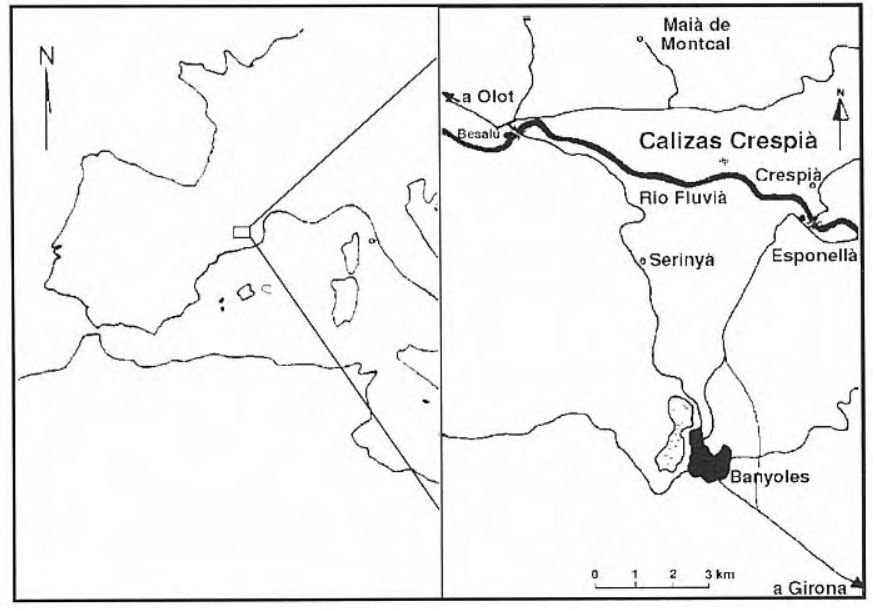

Figura 1. Localización geográfica del yacimiento de Crespià (Gerona).

Geographic location of the Crespià site (Gerona).

\section{INTRODUCCIÓN}

\section{SITUACIÓN GEOGRÁFICA Y HALLAZGO DE LOS RESTOS}

El yacimiento de Crespià se encuentra en las calizas de la Formación Incarcal, pertenecientes a un complejo fosilífero de especiales características geológicas situado en el noreste de España, en la provincia de Gerona (Figs. $1,2)$. Dichas calizas son de origen lacustre y se formaron durante el Plioceno final, abarcando una gran extensión de terreno. Es importante señalar que, entre los sedimentos de este yacimiento, se encuentran unos husos de arcillas de una edad del final del Pleistoceno inferior, de gran riqueza en fósiles de vertebrados, y que corresponden al complejo de yacimientos de Incarcal (Galobart et al., 1990; Maroto y Galobart, 1992; Galobart, 1996).

El yacimiento de Crespià es conocido por la abundancia de restos fósiles de vegetales (Villalta y Vicente, 1972; Roiron, 1983) y hasta el hallazgo del esqueleto de rinoceronte, objeto de este trabajo, la presencia de fósiles de vertebrados era nula. La localización de este esqueleto en Crespià fue, por tanto, un hecho excepcional en las calizas de la Formación Incarcal. Una visita rutinaria al conjunto de yacimientos de Incarcal permitió detectar, en la pared de un canal de desguace de la cantera, una serie de fragmentos de huesos que a la postre serían las costillas seccionadas del rinoceronte. La recuperación del fósil tuvo lugar en febrero de 1995 y cabe reseñar la realización de un molde de silicona del animal en conexión anatómica (Fig. 3). Una revisión preliminar de los restos hallados permitió a Galobart (1996) atribuirlos a Stephanorhinus etruscus Falconer, determinación que se confirma con el presente estudio sistemático. Stephanorhinus etruscus es el rinoceronte característico del Villafranquiense europeo, con una distribución bioestratigráfica desde la biozona MN16 hasta la MN19 (Guérin, 1980; Cerdeño, 1998). Fuera de España, está ampliamente representado y reemplaza a la especie rusciniense $S$. megarhinus De Christol; puntualmente, aparece asociado a $S$. jeanvireti Guérin, en algunos yacimientos del Villafranquiense inferior de Francia (Guérin, 1972; 1980). En España, S. etruscus, que sustituye a $S$. miguelcrusafonti Guérin y Santafé, característico de las biozonas MN 14 y 15, está representado en una decena de localidades (Santafé y Casanovas, 1987; Cerdeño, 1989a; 1993; Mazo, 1995; Mazo y Torres, 1990; Galobart, 1996; Alberdi et al.,1997). Es posible que restos pleistocenos, como los de Venta Micena, Cúllar de Baza y Huéscar-1, identificados como S. etruscus (Cerdeño, 1993), pudieran corresponder a S. hundsheimensis; no obstante, la confirmación de esta posibilidad no entra dentro de los objetivos de este trabajo. De acuerdo con Fortelius et al., (1993), S. etruscus sería sustituido por $S$. hundsheimensis $(=D$. etruscus brachycephalus sensu Fortelius et al., o.c.), característico de la parte inferior del Pleistoceno medio. El esquema filogenético propuesto por los autores citados vincula a $S$. etruscus y $S$. hundsheimensis con $S$. jeanvireti. Mazza et al., (1993) determinan como $S$. cf.

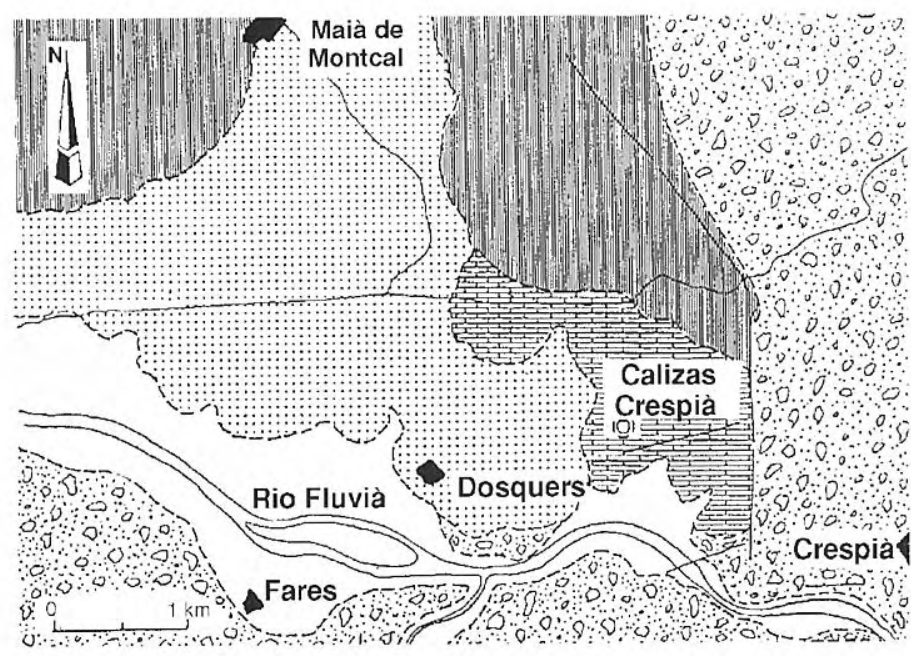

Figura 2. Marco geológico del área de Crespià. Geological map of the Crespià area.
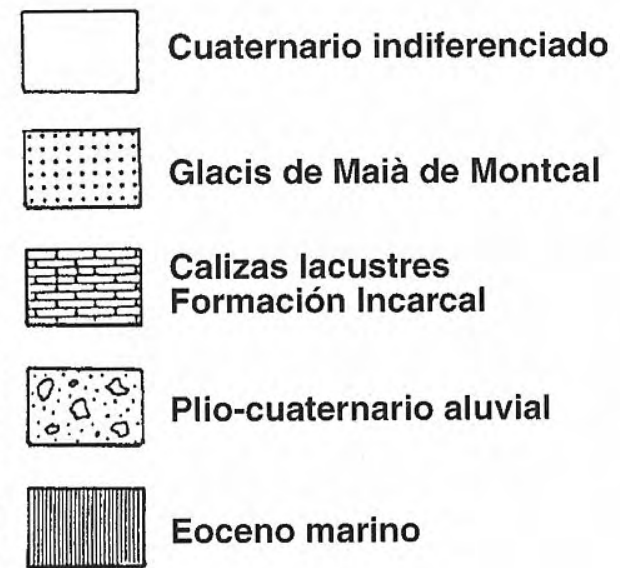

Plio-cuaternario aluvial

Eoceno marino 


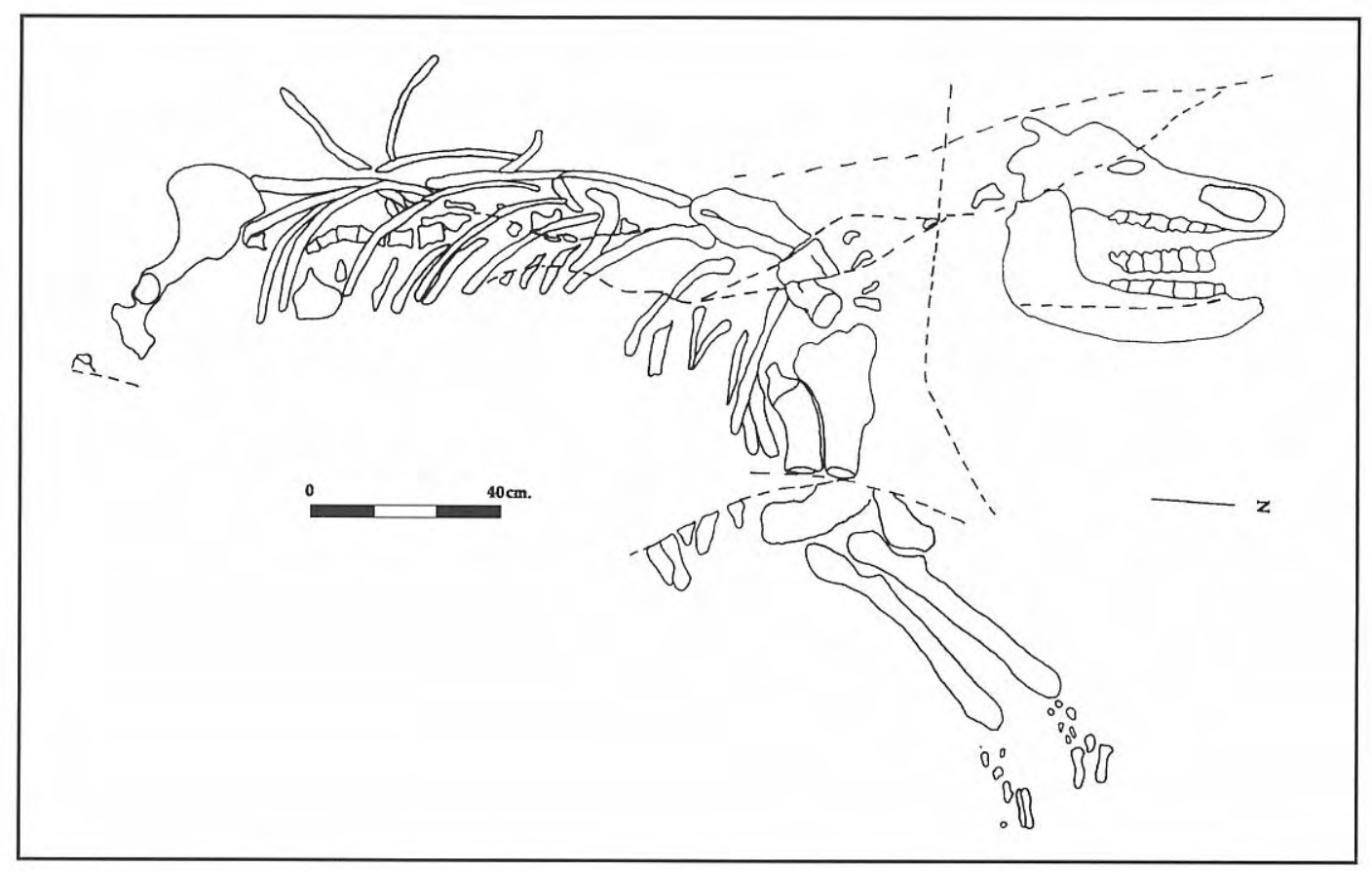

Figura 3. Dibujo esquemático de los restos del esqueleto de S. etruscus hallado en las calizas de Crespià. La líneas discontinuas marcan pequeñas fracturas en la roca.

Schematic drawn of S. etruscus skeleton from Crespià. The dashed lines show minor fractures in the sedimentary rock.

hundsheimensis los restos del Pleistoceno inferior tardío de Pietrafitta y postulan que los de otras localidades europeas de edad comparable - Pirro, Westerhovenpudieran constituir una forma de transición altamente variable en el ámbito de poblaciones locales. Fortelius et al., (o.c.) consideran esta posibilidad, aunque apuntan la hipótesis de que $S$. etruscus pudiera constituir un morfotipo más derivado cuya expresión última fuese $S$. hemitoechus puesto que en ambas especies están ausentes caracteres dentales concurrentes en S. hundsheimensis y S. jeanvireti.

E1 yacimiento de Crespià ocupa una posición intermedia entre las biozonas MN16 y MN17 (Fig. 4), lo que permite correlacionarlo, por ejemplo, con El Rincón1 (Albacete), en España, y Triversa en Italia.

\section{GEOLOGÍA REGIONAL Y LOCAL}

Los yacimientos de la Formación Incarcal están situados en la Cuenca lacustre de Banyoles-Besalú, que ocupa una franja deprimida, orientada de Norte a Sur, situada entre las de relieves levantados de la Garrotxa, de edad eocena, y la depresión del Empordà, de materiales mayoritariamente neógenos (Figs. 1 y 2).

La cuenca lacustre ha evolucionado desde el final del Terciario y durante el Cuaternario. En ella se distinguen tres unidades geomorfológicas que son, de Norte a Sur: el glacis de Maià de Montcal, el Pla de l' Usall y la Cubeta lacustre de Banyoles (Julià, 1980). El yacimiento de Crespià se encuentra en la primera de estas tres unidades y su sustrato mayoritario está formado por las calizas lacustres de la Formación Incarcal (Julià, 1980), que según Roiron (1983), a partir de los restos vegetales, pueden ser de una edad de finales del Plioceno.

Las calizas de Incarcal están constituidas de forma mayoritaria por calcisiltitas y calcilutitas, con capas de acumulación de fragmentos de carofíceas. Tienen un color totalmente blanco, con un contenido de $\mathrm{CaCO}_{3}$ próximo al $100 \%$, y están muy poco consolidadas. Contienen gran cantidad de restos vegetales, tanto hojas (Villalta y Vicente, 1972; Roiron, 1983) como polen (Geurts, 1977, 1979; Suc,

\begin{tabular}{|c|c|c|c|c|}
\hline Epoca & \multicolumn{2}{|c|}{$\begin{array}{l}\text { Edades basadas en } \\
\text { micromamiferos } / \mathrm{Pisos} \\
\text { continentales }\end{array}$} & España & Otras localidades \\
\hline $\begin{array}{l}\text { Pleistoceno } \\
\text { medio }\end{array}$ & \multicolumn{2}{|c|}{ Toringiense } & Cúllar de Baza-1 & $\begin{array}{l}\text { Mosbach-2 } \\
\text { Isernia* }\end{array}$ \\
\hline \multirow{2}{*}{$\begin{array}{l}\text { Pleistoceno } \\
\text { inferior }\end{array}$} & \multicolumn{2}{|c|}{$\begin{array}{l}\text { Bihariense } \\
\text { superior }\end{array}$} & Huéscar-1 & $\begin{array}{c}\text { Le Vallonet* } \\
\text { Cesi }\end{array}$ \\
\hline & $\begin{array}{l}\text { Bihariense } \\
\text { inferior }\end{array}$ & $\begin{array}{l}\text { Vilafranquiense } \\
\text { superior }\end{array}$ & $\begin{array}{l}\text { Cueva Victoria } \\
\text { Incarcal } \\
\text { Venta Micena }\end{array}$ & $\begin{array}{l}\text { Capitone } \\
\text { Chilhac } \\
\text { Valdarno }\end{array}$ \\
\hline \multirow[b]{2}{*}{$\begin{array}{l}\text { Plioceno } \\
\text { superior }\end{array}$} & $\begin{array}{c}\text { Villaniense } \\
\text { superior } \\
\text { MN } 17\end{array}$ & $\begin{array}{c}\text { Villafranquiense } \\
\text { medio }\end{array}$ & $\begin{array}{l}\text { Puebla de } \\
\text { Valverde } \\
\text { Crespià } \\
\text { Rincón-1 }\end{array}$ & \multirow{2}{*}{$\begin{array}{l}\text { Cornillet } \\
\text { Triversa } \\
\text { Viallette } \\
\text { Etouaires }\end{array}$} \\
\hline & $\begin{array}{c}\begin{array}{c}\text { Villaniense } \\
\text { inferior }\end{array} \\
\text { MN } 16\end{array}$ & $\begin{array}{l}\text { Villafranquiense } \\
\text { inferior }\end{array}$ & $\begin{array}{c}\text { Huélago } \\
\text { Villaroya } \\
\text { Las Higueruelas }\end{array}$ & \\
\hline
\end{tabular}

Figura 4. Edad de las localidades europeas con S. etruscus y $S$. hundsheimensis $(*)$ citadas en el texto y en las tablas.

Age of the European localities with S. etruscus and $\mathrm{S}$. hundsheimensis (*) mentioned in text and tables. 


\begin{tabular}{|l|c|c|c|c|}
\cline { 2 - 5 } \multicolumn{1}{c|}{} & Crespià & \multicolumn{3}{c|}{ Guérin, 1980) } \\
\cline { 2 - 5 } \multicolumn{1}{c|}{} & & Mínima & Media & Máxima \\
\hline Dist. cóndilo-extremo nasal & 598 & 530 & 615,0 & 648 \\
\hline Dist. vértice occipital-extremo nasal & 620 & 530 & 620,6 & 655 \\
\hline Longitud escotadura nasal & 183 & 138 & 191,7 & 209 \\
\hline Dist. escotadura nasal-inicio órbita & 99,9 & 90 & 105,5 & 123 \\
\hline Dist. borde post. M3-cóndilo occipital & 300 & 252 & 290,6 & 320 \\
\hline Dist. extremo nasal-inicio de la órbita & 283 & 236 & 298,4 & 327 \\
\hline Altura del cráneo por encima del P2 & 114,1 & 124 & 159,5 & 178 \\
\hline
\end{tabular}

Tabla 1. Dimensiones craneales de S. etruscus de Crespià comparadas con los datos de Guérin (1980).

Comparative dimensions of the skull of $\mathrm{S}$. etruscus from Crespià and some French and Italian localities (after Guérin, 1980).

1982). Estas calizas están muy fracturadas y basculadas. Se aprecian discordancias angulares y algún slump. Las fallas, directas, son muy abundantes y algunos autores ven en ellas los indicios de una actividad paleosismológica (Fleta et al., 1996). La potencia total de la serie que aflora en la cantera es de unos 70 m (Julià y Villalta, 1984).

\section{MATERIAL Y METODOLOGÍA}

Los restos estudiados pertenecen a la colección de Paleontología de Vertebrados del Museu Comarcal d'Arqueologia de Banyoles, aunque en el momento de su estudio se hallaban temporalmente depositados en el Institut de Paleontologia M. Crusafont de Sabadell. Cada ejemplar está identificado con la sigla CC seguida de un número. Algunos elementos esqueléticos (cráneo, Mcs III) están alterados por compresión diagenética. Otros huesos (radio, ulna y coxal), muy deteriorados, han sido medidos sobre los negativos de poliéster obtenidos a partir del molde de silicona realizado "in situ".

El estudio comparativo se ha basado principalmente en los trabajos de Guérin (1980), Mazza (1988) y Fortelius et al., (1993). Los datos del material comparado son básicamente bibliográficos, pero también se incluyen datos inéditos de algunos ejemplares de La Puebla de Valverde depositados en el Institut de Paleontologia M. Crusafont de Sabadell.

Las abreviaturas utilizadas en el texto y las tablas corresponden a: CC (Calizas de Crespià); CV (Cueva Victoria); Hu (Huéscar-1); IN (Incarcal); PV (La Puebla de Valverde); VM (Venta Micena); IPS (Institut de Paleontologia M. Crusafont); Anch. (anchura); Anat. (anatómica); art. (articular); DAP (diámetro anteroposterior); di. (diáfisis); dis. (distal); Dist.(distancia); DT (diámetro transverso); L o Long (longitud); H (altura); inf (inferior); $\mathrm{P} / \mathrm{p}$ (premolar superior/inferior); $\mathrm{M} / \mathrm{m}$ (molar superior/inferior); Máx (máximo); Mín (mínimo); prox. (proximal); sup. (superior).

Los valores numéricos que se citan entre paréntesis, tanto en el texto como en las tablas, corresponden a aquellas medidas que son de difícil obtención o bien que la deformación del fósil sólo permite una aproximación a su valor real.

\section{PALEONTOLOGÍA SISTEMÁTICA}

\author{
ORDEN PERISSODACTYLA Owen, 1848 \\ Familia Rhinocerotidae Owen, 1845 \\ Subfamilia Rhinocerotinae Owen, 1845 \\ Tribu Rhinocerotini Owen, 1845 \\ Stephanorhinus Kretzoi, 1942
}

\section{Stephanorhinus etruscus (Falconer, 1868)}

\section{DESCRIPCIÓN COMPARATIVA \\ Cráneo (CC-1; Tabla 1; Fig. 5a)}

El cráneo está completo, pero comprimido lateralmente y desplazado dorsoventralmente en la mitad posterior. De las medidas que se han podido tomar, la altura por encima del P2 no alcanza el mínimo específico (Guérin, 1980 -"D. etruscus etruscus"-) y superarían los máximos las que relacionan la distancia del vértice occipital-proceso postorbitario y la longitud de la fosa órbito-temporal, lo que indicaría un neurocráneo relativamente largo respecto al rostro.

Las distancias vértice occipital-extremo del nasal y borde posterior del M3-cóndilo occipital coinciden con los valores medios.

El cráneo es dolicocéfalo, con perfil dorsal cóncavo y elevación del occipital en ángulo estimado inferior a $165^{\circ}$. La base para el cuerno nasal es una cúspide masiva, menos rugosa que en $S$. hundsheimensis Toula de Isernia La Pineta (Italia; Sala y Fortelius, 1993, Lám. 1) y menos relevante que la observada en "D." etruscus de Chilhac (Francia; Boeuf, 1994, Lám. 52). En el cráneo de Capitone (Italia), atribuido a una hembra por Ambrosetti (1972), la base de sustentación se manifiesta como un leve abultamiento. La base del cuerno nasal dista $45 \mathrm{~mm}$ del extremo rostral; la del frontal es difusa y sólo algo rugosa lateralmente. Mazza (1988) indica diferencias en el grado de señalización de la base para el cuerno posterior, desde bien desarrollada a apenas distinguible. Mazo (1995) no la observa en el cráneo de Las Higueruelas (Ciudad Real) y sí lo hace Boeuf (1994) en el de Chilhac. Hay un foramen infraorbital grande, ovalado y situado al nivel de P4-M1. La posición del fondo de la escotadura nasal -mitad anterior del P4- coincide con lo expuesto por Guérin (1980) y Fortelius et al.,(1993) para $S$. etruscus. Comparando con S. hundsheimensis, la escotadura nasal alcanza el mismo nivel en Mosbach (Schroeder, 1903, Tabla 1, Lám. 1a) y se retrasa en Isernia (Sala y Fortelius, 

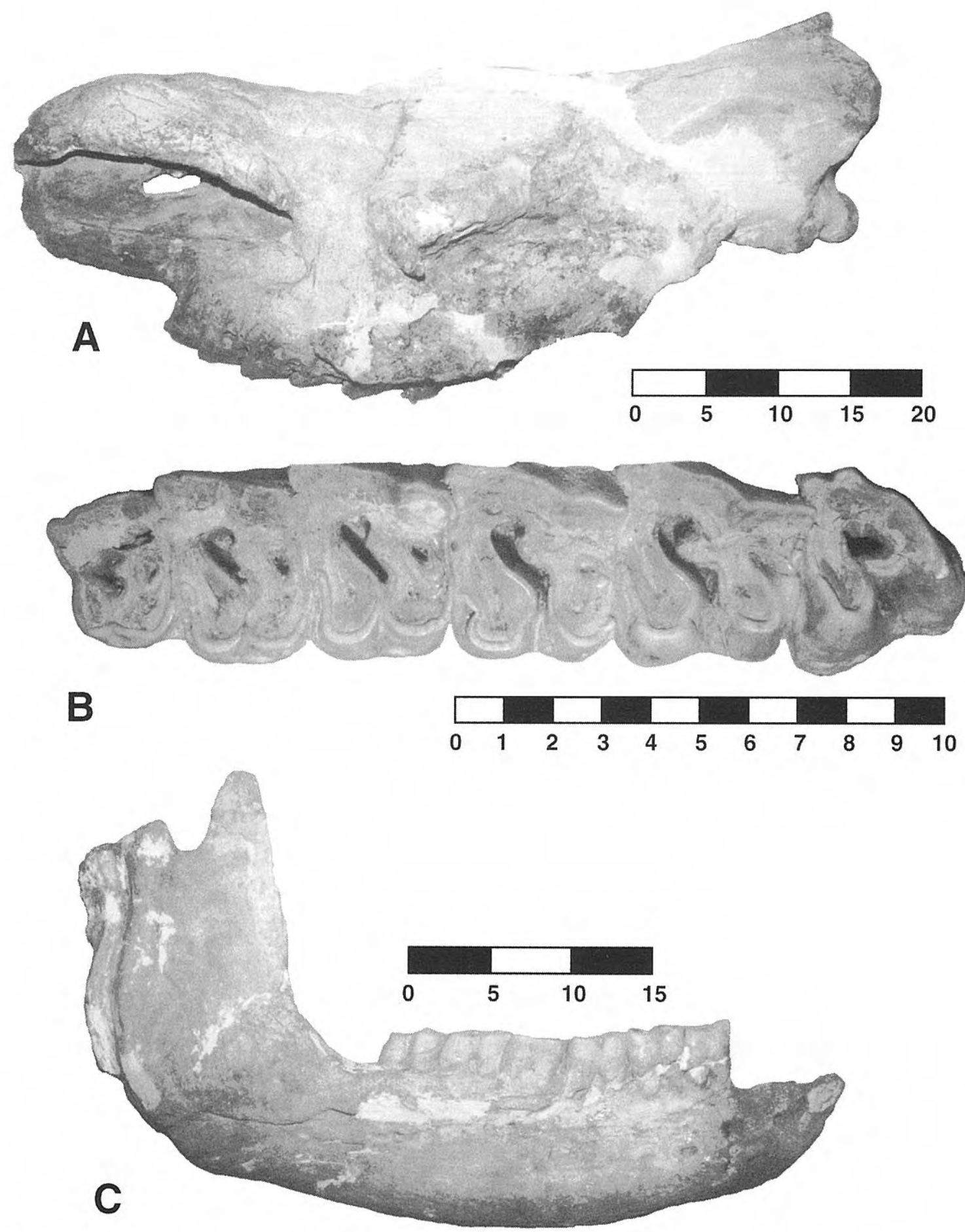

Figura 5. S. etruscus de Crespià. a. Cráneo; vista latero-superior. b. Serie superior izquierda P2-M3; vista oclusal. c. Hemimandíbula derecha; vista labial.

S. etruscus from Crespià. a. Skull, latero-superior view. b. Left upper series P2-M3, occlusal view. c. Right hemimandible, labial view.

1993). El borde anterior de la órbita, sobre la parte posterior del M2, se adelanta respecto a $S$. hundsheimensis de Isernia (Sala y Fortelius, o.c.) y al promedio de $S$. etruscus (Guérin, o.c. y Fortelius et al., o.c.) aunque coincide con Capitone (Ambrosetti, 1972), Las Higueruelas (Mazo, 1995) y Senèze -nº-96129- (en Guérin, o.c. Lám. 11E), en un cráneo de esta localidad, el borde orbitáreo está más adelantado (Bouchud, 1966, Lám. 8).
Los arcos cigomáticos, insertados sobre el intervalo M2M3, presentan yugales altos y de sección rectangular y escamosos ovalados, delgados y ventralmente recorridos por una acanaladura para el tendón asociado al músculo masetero.

La apófisis postglenoidea es robusta e inclinada hacia delante como corresponde a la especie (Guérin, 1980); la postimpánica es más corta y se une a la anterior cerrando 


\begin{tabular}{|l|c|c|c|c|}
\cline { 2 - 5 } \multicolumn{1}{c|}{} & Crespià & \multicolumn{3}{c|}{ Guérin, 1980) } \\
\cline { 2 - 5 } \multicolumn{1}{c|}{} & & Mínima & Media & Máxima \\
\hline Longitud sínfisis-borde post. talón & 457 & 430 & 476,42 & 509 \\
\hline Altura de la rama horizontal entre p2-p3 & $(71,2)$ & 55,5 & 66,32 & 80 \\
\hline Altura de la rama horizontal entre p3-p4 & $(77,6)$ & 62,5 & 71,94 & 83,5 \\
\hline Altura de la rama horizontal entre p4-m1 & $(90,4)$ & 64 & 76,23 & 85,5 \\
\hline Altura de la rama horizontal entre m1-m2 & $(91,9)$ & 65 & 82,79 & 91 \\
\hline Altura de la rama horizontal entre m2-m3 & $(94,5)$ & 70 & 85,87 & 96,5 \\
\hline Altura de la rama horizontal post. m3 & $(94,2)$ & 79 & 90,78 & 105 \\
\hline DAP de la rama vertical (sup.oclusal) & 128,2 & 115 & 143,03 & 152 \\
\hline
\end{tabular}

Tabla 2. Dimensiones de la mandíbula de S. etruscus de Crespià, comparadas con los datos de Guérin (1980).

Comparative dimensions of the mandible of $\mathrm{S}$. etruscus from Crespià and some Spanish, French, Italian and Germany localities (after Guérin, 1980).

inferiormente el meato auditivo externo. Proceso paraoccipital dirigido hacia delante. La cara occipital, trapezoidal, es más baja que en Las Higueruelas $(140 \mathrm{~mm}$ frente a $165 \mathrm{~mm}$ ) y similar a la de algunos cráneos de Valdarno superior (Italia; Mazza, 1988). Los cóndilos son pequeños. En el basioccipital, algo incompleto, es visible la escotadura esfeno-espinosa y un pequeño foramen condiliano accesorio circular.

\section{Mandíbula (CC-2; Tabla 2; Fig. 5c)}

La sínfisis es corta, con el borde posterior a la altura del valle anterior del $\mathrm{p} 2$, adelantado respecto a la media que señala Guérin (1980) para S. etruscus, aunque ajustado a los límites de la variabilidad. El foramen principal es elíptico, profundo, dista $30 \mathrm{~mm}$ del margen alveolar y se sitúa bajo el lóbulo anterior del p2; entre p2 y p3 hay un foramen accesorio. Las ramas horizontales son cortas y delgadas, con perfil ventral ligeramente convexo y el labial aplanado en la serie premolar. Su altura, entre p4 y m2, se encuentra alterada por compresión diagenética. En la cara lingual, una concavidad recorre longitudinalmente su parte central. El talón es redondeado y poco saliente. Las ramas ascendentes muestran una ligera inclinación hacia atrás del borde anterior, tal como corresponde a la especie (Guérin, 1980). Los cóndilos, incompletos, son robustos, triangulares y dispuestos en ángulo de $230^{\circ}$. La incisión sigmoidea es poco profunda.

La impresión muscular del pterigoideo medial es difusa, ovalada y está situada simétricamente respecto a la fosa masetérica que está delimitada inferiormente por el área de inserción del tendón intermedio.

Métricamente, las ramas ascendentes están dentro de la variabilidad señalada por Guérin (1980), pero son más bajas que en los ejemplares de Valdarno superior (Mazza, 1988) y Las Higueruelas (Mazo, 1995), los cuales, a su vez, presentan sínfisis más largas.

Dentición superior (Tabla 3; Fig. 5b)

No hay alvéolos para la dentición anterior. En la serie yugal, el esmalte se estría verticalmente en el tercio inferior de los dientes. Hay una ligera crenulación en la pared lingual del gancho en P3 y M3. Esta peculiaridad se aprecia en un M3 de Huéscar-1 (Granada; Mazo et al., 1985, Lám. II) y en un M1 de Villarroya (La Rioja; Villalta, 1952).

En los premolares, los pliegues del parastilo y del paracono se acentúan gradualmente de P2 a P4. El del metacono se insinúa en P3 y se señaliza bien en P4, no así en su homólogo de Incarcal -IN-I-740- (Galobart, 1996) donde no se desarrolla. El valle medio forma una foseta central triangular en P2 y con forma de "L" en P3 y P4. Los ganchos son pequeños. En el P4 hay una pequeña crista y un surco vertical anterior recorre el protocono. El cíngulo mesial es alto y grueso en P3 y P4; el lingual aparece débilmente a la entrada del valle medio y en la mitad anterior del hipocono.

Comparando con las dimensiones aportadas por Guérin (1980) para S. etruscus (“D. etruscus etruscus"), se observa que la anchura se mantiene en toda la serie por encima de los mínimos específicos; la longitud, en cambio, se aproxima gradualmente a éstos entre P2 y P3 y se sitúa por debajo en el P4.

Individualmente, los $\mathrm{P} 2$ y $\mathrm{P} 3$ de Crespià son más estrechos y largos que los del maxilar "A"de Chilhac (Boeuf, 1994), similares a los de Venta Micena IPSVM15.567 (Granada; Santafé y Casanovas, 1987) y más cortos que los de Huéscar-1 (Cerdeño, 1989a) y Cueva Victoria (Tabla 3).

En los molares, el pliegue del metacono está marcado en M1, a diferencia de lo observado en Huéscar-1 (Santafé y Casanovas, 1987; Cerdeño, 1989a) y Venta Micena (Santafé y Casanovas, 1987); hay mesostilo en M2 y leve pinzamiento del parastilo en M3. E1 protocono aparece estrangulado en M1 y M2; en M3, con menos desgaste, sólo se ve un surco vertical anterior en la mitad inferior. Gancho redondeado en M1 y más estilizado en los otros dos molares. En M3 hay crista. En M1 y M2 hay restos de cíngulo a la entrada del valle medio y en la pared interna del hipocono de M1.

La longitud de los molares está por debajo del mínimo específico (Guérin, 1980). La anchura es algo superior sólo en M2. Los molares de Crespià son más cortos y en general más estrechos que los de Huéscar 1 y Venta Micena (Tabla 3).

Dentición inferior (Tabla 4)

La serie carece de p1. La longitud respectiva de las series premolar y molar es de $92,7 \mathrm{~mm}$ y de $118 \mathrm{~mm}$. La primera supera más netamente el mínimo de $S$. etruscus (" $D$. etruscus etruscus") señalado por Guérin (1980, Tabla 90). El índice 100 x L serie molar / L p3-p4 $(180,82)$ coincide con el mínimo específico. La longitud relativa de los dos últimos premolares $(179,6)$ es inferior a la mínima calculada a partir de Guérin (1980, Tabla 89).

El protocónido es saliente en p4, m1 y m2. El hipocónido se hace más anguloso de $\mathrm{m} 1 \mathrm{a}$ m3. El p2 carece de cíngulos 


\begin{tabular}{|c|c|c|c|c|c|c|c|c|c|c|c|c|}
\hline & & Crespià & \multicolumn{4}{|c|}{ Venta Micena (2) } & \multicolumn{5}{|c|}{ Huéscar-1 } & $\mathrm{CV}$ \\
\hline $\mathrm{P} 2$ & $\begin{array}{l}\mathrm{L} \\
\mathrm{A} \\
\end{array}$ & \begin{tabular}{|l|}
31,1 \\
37,4 \\
\end{tabular} & $\begin{array}{c}(29,5) \\
- \\
\end{array}$ & & & & $\begin{array}{l}33,5 \\
34,9 \\
\end{array}$ & $\begin{array}{l}33,2 \\
36,2 \\
\end{array}$ & & & & \\
\hline P3 & $\begin{array}{l}\mathrm{L} \\
\mathrm{A}\end{array}$ & $\begin{array}{l}34,9 \\
46,8 \\
\end{array}$ & $\begin{array}{r}40 \\
- \\
\end{array}$ & $\begin{array}{l}37 \\
46 \\
\end{array}$ & & & $\begin{array}{l}41,3 \\
53,4 \\
\end{array}$ & & & & & \\
\hline P4 & $\begin{array}{l}\mathrm{L} \\
\mathrm{A}\end{array}$ & $\begin{array}{l}36,1 \\
50,6\end{array}$ & $\begin{array}{l}(37,3) \\
(49,9)\end{array}$ & & & & $\overline{60}$ & $\begin{array}{l}(>42) \\
(54,5)\end{array}$ & $\begin{array}{c}(>43,5) \\
-\end{array}$ & $\begin{array}{l}(42)^{(2)} \\
(54)^{(2)}\end{array}$ & $54,4^{(1)}$ & $\begin{array}{l}(41)^{(2)} \\
(51)^{(2)}\end{array}$ \\
\hline M1 & $\begin{array}{l}\mathrm{L} \\
\mathrm{A}\end{array}$ & $\begin{array}{l}39,0 \\
48,2\end{array}$ & $\begin{array}{c}(45) \\
(51,2)\end{array}$ & $\begin{array}{l}42 \\
58\end{array}$ & & & $\begin{array}{l}52 \\
53\end{array}$ & $\begin{array}{l}(49) \\
51,7\end{array}$ & $\begin{array}{l}48,7 \\
55,6\end{array}$ & $\begin{array}{l}48,6 \\
(55)\end{array}$ & $\begin{array}{c}49 /(43)^{(1)} \\
52 / 51 / 50^{(1)}\end{array}$ & \\
\hline M2 & $\begin{array}{l}\mathrm{L} \\
\mathrm{A}\end{array}$ & $\begin{array}{l}42,9 \\
50,4\end{array}$ & $\overline{56}$ & $\begin{array}{l}51 \\
-\end{array}$ & $\begin{array}{c}50,5 \\
49\end{array}$ & $\begin{array}{l}49 \\
-\end{array}$ & $\begin{array}{c}53,5^{(2)} \\
56^{(2)}\end{array}$ & & & & & $\begin{array}{l}47^{(2)} \\
52^{(2)}\end{array}$ \\
\hline M3 & $\begin{array}{r}\text { L } \\
\text { A } \\
\text { Ana. }\end{array}$ & $\begin{array}{c}44,5 \\
45,2 \\
34\end{array}$ & $\begin{array}{c}49 \\
48 \\
-\end{array}$ & & & & $\begin{array}{c}(42) \\
- \\
-\end{array}$ & $\begin{array}{c}46,4^{(1)} \\
50,6^{(1)} \\
49^{(1)}\end{array}$ & & & & \\
\hline
\end{tabular}

Tabla 3. Dimensiones de la dentición superior de S. etruscus de Crespià comparadas con los datos de Cerdeño (1989a); (1) Mazo et al., (1985); (2) Santafé y Casanovas (1987).

Comparative dimensions of the upper teeth of $\mathrm{S}$. etruscus from Crespià and some Spanish localities [after Cerdeño, 1989a; (1) Mazo et al., 1985; (2) Santafé and Casanovas (1987)].

laterales: el labial es fuerte en p4 y en los molares, donde desciende sobre el lóbulo anterior, sin alcanzar la base del surco de separación; hay restos de cíngulo en la base del lóbulo posterior en p3 y p4. La fosa del trigónido está cerrada en $\mathrm{m} 1$ dado su mayor desgaste; la del talónido está por debajo de la anterior en p3 (unos $3 \mathrm{~mm}$ ) y en $\mathrm{m} 2$ (unos 5 $\mathrm{mm}$ ). El perfil de los valles en $\mathrm{m} 2$ y $\mathrm{m} 3$ es en "V".

Al igual que en la serie superior, la longitud de los premolares apenas alcanza el mínimo establecido por Guérin (1980). La anchura, en cambio, supera en p3 y p4 el valor medio. Comparativamente, el p2 es más estrecho y sobre todo más corto que sus homólogos de Huéscar-1. Por el contrario, los p3 y los p4 de Crespià y Huélago son proporcionalmente más anchos que los de Venta Micena (Tabla 4).

En $\mathrm{m} 1$ y $\mathrm{m} 2$, las dimensiones rondan los mínimos específicos de $S$. etruscus ("D. etruscus etruscus") indicados por Guérin (1980, Tabla 89), siendo algo inferiores en m3. Los molares de Crespià son proporcionalmente más anchos y cortos que los de Huéscar-1, Huélago y Venta Micena (Tabla 4).

\section{Esqueleto postcraneal}

Escápula (CC-3). La nula señalización del cuello y el desarrollo del tubérculo supraglenoideo y de la apófisis coracoides coinciden con S. cf. hundsheimensis de Pirro (Italia; Mazza et al.,1993, Lám. 6, Fig. 4) y difieren de $S$. etruscus (Ambrosetti, 1972, Fig. 2; Fortelius et al.,1993, Lám. 1, Fig 1a). Los diámetros de la cavidad glenoidea (70 / $>60$ ) son superiores a los de S. etruscus (Fortelius et al., o.c. Tabla 10B) e iguales a los de $S$. cf. hundsheimensis de Pietrafitta (Mazza et al.,1993. Tabla 3). Respecto a esta localidad, el DT distal en Crespià es ligeramente superior (115 frente a 110).

Húmero. Se conserva un fragmento (CC-4) que comprende la articulación proximal y $2 / 3$ de la diáfisis. La diáfisis es de sección subtriangular, con el vértice en posición anterointerna. El surco de separación de la tróclea es estrecho y profundo. Hay una débil impresión del ligamento colateral medio del codo. El DT proximal supera el valor medio aportado por Guérin (1980, Tabla 91) para $S$. etruscus ("D. etruscus etruscus"). Los diámetros de la diáfisis entran en los límites de variación de la especie,

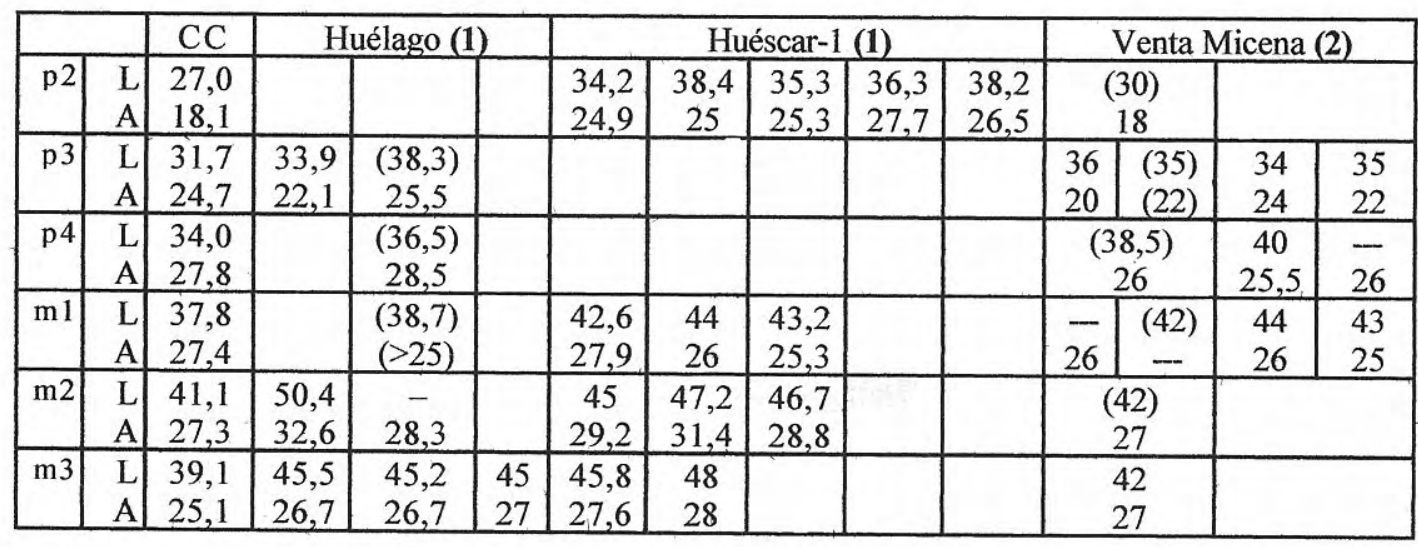

Tabla 4. Dimensiones de la dentición inferior de S. etruscus de Crespià. (1) Cerdeño (1989a); (2) Santafé y Casanovas (1987). Comparative dimensions of the lower teeth of $\mathrm{S}$. etruscus from Crespià and some Spanish localities [after (1) Cerdeño, 1989a; (2) Santafé and Casanovas, 1987]. 


\begin{tabular}{|c|c|c|c|c|c|c|c|c|c|c|}
\hline & & \multicolumn{2}{|c|}{ Art. prox } & \multicolumn{2}{|c|}{ Diafisis } & \multicolumn{2}{|c|}{ Ep. Distal } & \multicolumn{2}{|c|}{ Art. distal } \\
\hline & & Long. & DT & DAP & DT & DAP & DT & DAP & DT & DAP \\
\hline Crespià & & 350 & (68) & (49) & 39 & $(40)$ & 74,5 & 54,2 & 67 & $(33,2)$ \\
\hline Puebla deVa & lverde (1) & $\begin{array}{l}415 \\
407\end{array}$ & $\overline{86}$ & $\begin{array}{l}60 \\
58\end{array}$ & $\begin{array}{l}54 \\
49\end{array}$ & $\begin{array}{l}40 \\
41\end{array}$ & $\begin{array}{l}81 \\
85\end{array}$ & $\begin{array}{l}69 \\
58\end{array}$ & & \\
\hline $\begin{array}{l}\text { St. Vallier } \\
\text { (1) }\end{array}$ & $\begin{array}{l}\text { Min. } \\
\text { Media } \\
\text { Max. }\end{array}$ & $\begin{array}{c}386 \\
386,5 \\
387\end{array}$ & $\begin{array}{c}84 \\
88,2 \\
91\end{array}$ & $\begin{array}{c}55 \\
57,6 \\
60\end{array}$ & $\begin{array}{l}42 \\
48 \\
52\end{array}$ & $\begin{array}{c}32 \\
35,1 \\
39\end{array}$ & $\begin{array}{c}86 \\
87,5 \\
89\end{array}$ & $\begin{array}{c}58 \\
59,5 \\
61\end{array}$ & & \\
\hline Senèze (1) & $\begin{array}{l}\text { Min. } \\
\text { Media } \\
\text { Max. }\end{array}$ & $\begin{array}{c}342 \\
362,2 \\
382\end{array}$ & $\begin{array}{c}82 \\
84,5 \\
87\end{array}$ & $\begin{array}{c}58 \\
61 \\
59,3\end{array}$ & $\begin{array}{c}40 \\
45,3 \\
50\end{array}$ & $\begin{array}{c}30 \\
34,8 \\
39\end{array}$ & $\begin{array}{c}86 \\
89,7 \\
94\end{array}$ & $\begin{array}{c}60 \\
61,9 \\
63\end{array}$ & & \\
\hline $\begin{array}{l}\text { Venta } \\
\text { Micena (2) }\end{array}$ & $\begin{array}{l}\text { CL98-5 } \\
\text { CI-458 }\end{array}$ & - & $\begin{array}{c}74 \\
(80)\end{array}$ & $\begin{array}{l}46,5 \\
(43)\end{array}$ & $\begin{array}{l}(40) \\
50\end{array}$ & $\begin{array}{l}\text { (37) } \\
\text { (34) }\end{array}$ & $\begin{array}{l}- \\
-\end{array}$ & $\begin{array}{l}- \\
-\end{array}$ & $\begin{array}{l}- \\
-\end{array}$ & $\begin{array}{l}- \\
-\end{array}$ \\
\hline $\begin{array}{l}\text { S. etruscus } \\
\text { (3) }\end{array}$ & $\begin{array}{l}\text { Min. } \\
\text { Media } \\
\text { Max. }\end{array}$ & $\begin{array}{c}342 \\
373,55 \\
415\end{array}$ & $\begin{array}{c}77,5 \\
86,14 \\
94\end{array}$ & $\begin{array}{c}50,5 \\
57,49 \\
65\end{array}$ & $\begin{array}{c}40 \\
47,57 \\
56\end{array}$ & $\begin{array}{c}30 \\
36,04 \\
42\end{array}$ & $\begin{array}{c}79 \\
86,92 \\
93,5\end{array}$ & $\begin{array}{c}49 \\
57,38 \\
63\end{array}$ & $\begin{array}{c}73,5 \\
74,83 \\
76,5\end{array}$ & $\begin{array}{c}38,5 \\
39,83 \\
41\end{array}$ \\
\hline $\begin{array}{l}\text { S. etruscus } \\
\text { (4) }\end{array}$ & $\begin{array}{l}\text { Min. } \\
\text { Media } \\
\text { Max. }\end{array}$ & $\begin{array}{c}354 \\
371,5 \\
386\end{array}$ & $\begin{array}{c}(65) \\
82,6 \\
89\end{array}$ & $\begin{array}{c}(40,2) \\
54,1 \\
68,2\end{array}$ & $\begin{array}{c}41 \\
46,0 \\
55\end{array}$ & $\begin{array}{c}27 \\
33,3 \\
37\end{array}$ & $\begin{array}{c}(71) \\
84,1 \\
90\end{array}$ & $\begin{array}{c}47 \\
52,4 \\
60\end{array}$ & $\begin{array}{c}53 \\
66,3 \\
74\end{array}$ & $\begin{array}{c}37 \\
39,7 \\
42\end{array}$ \\
\hline
\end{tabular}

Tabla 5. Dimensiones comparadas del radio de S. etruscus de Crespià. (1) Guérin y Heintz (1971); (2) Santafé y Casanovas (1987); (3) Guérin (1980); (4) Fortelius et al., (1993).

Comparative dimensions of the radius of $\mathrm{S}$. etruscus from Crespià and some Spanish, French and Italian localities [after (1) Guérin and Heintz, 1971; (2) Santafé and Casanovas, 1987; (3) Guérin, 1980; (4) Fortelius et al., 1993].

aunque el DT tiende al mínimo y el DAP al máximo. El DT coincide con el del ejemplar del Pozo de Piedrabuena (Ciudad Real; Mazo y Torres, 1990) y es inferior al de La Puebla de Valverde (Guérin y Heintz, 1971) y aún más respecto a Huéscar-1 (Cerdeño, 1989a). Las dimensiones que han podido tomarse son: DT prox., $160 \mathrm{~mm}$; DT cabeza articular, $71 \mathrm{~mm}$; DT a nivel de la tuberosidad deltoidea, (92) mm; DT diáfisis, 53,7 mm; DAP diáfisis, 65,7 mm; H ant. surco troclea, $(49,4) \mathrm{mm}$.

Radio (Tablas 5-6; Fig. 6a). El ejemplar CC-5 ostenta la mayor gracilidad de las calculadas (Tabla 6), para una longitud inferior a la media indicada para S. etruscus ("D. etruscus etruscus") por Guérin (1980, Tabla 92) y a la mínima dada por Fortelius et al., (1993, Tabla 12B). El DAP en la diáfisis es menor en el Pleistoceno inferior de Venta Micena y Voigstedt (Santafé y Casanovas, 1987). El perímetro proximal difiere del figurado por Guérin (1980, Fig. 61) por el ángulo más redondeado que forman las caras anterior e interna. La faceta proximal externa es profunda; la interna, aplanada y con DT de $28 \mathrm{~mm}$. La tuberosidad lateral no alcanza el borde articular. En la epífisis distal, la cresta externa que delimita el surco para el tendón extensor es más oblicua y masiva para facilitar la inserción del ligamento colateral lateral del carpo. La faceta para el escafoides es subtrapezoidal, extendida posteriormente e inclinada externamente (DT máx.: $12 \mathrm{~mm}$ ). La del semilunar es cóncava anteroposteriormente, tiene forma de triángulo rectángulo y está recorrida transversalmente por un surco (H: $10 \mathrm{~mm})$.

Ulna (CC-6; Tabla 7; Fig. 6a). El perfil externo de la epífisis proximal es igual al de Senèze y Valdarno superior (Guérin, 1980, Fig. 62; Fortelius et al., 1993, Lám. 3). El olécranon presenta una inclinación posterior en ángulo de $120^{\circ}$ y se ensancha gradualmente a la par que se curva internamente. La superficie medial es lisa y la externa rugosa proximalmente para proporcionar inserción al tríceps braquial, al tendón del antebrazo y al ancóneo lateral y caudalmente. La faceta para el piramidal es más cóncava transversalmente que convexa anteroposteriormente. La escotadura troclear es más baja que en La Puebla de Valverde (PV 040; (?) $47 \mathrm{~mm}$ frente a $53 \mathrm{~mm}$ ), más convexa y está dividida por una arista más roma.

Figura 6. Esqueleto postcraneal de $S$. etruscus de Crespià. a. Ulna y radio derechos en conexión; vista lateral. b. Huesos del carpo derecho (escafoides, semilunar y piramidal); vista proximal. c. Pisiforme derecho; vista anterior. d. Trapezoides derecho; vista anterior. e. Magno derecho; vista anterior. f. Unciforme derecho; vista anterior. g. Primera falange lateral; vista posterior; la flecha indica una posible patología. h. Metacarpos de S. etruscus de La Puebla de Valverde (izquierda) y Crespià (derecha): h1, Mc II, vistas medial y proximal; h2, Mc III, vistas anterior y proximal; h3, Mc IV, vistas medial y proximal.

S. etruscus from Crespià. a. Right ulna and radius, lateral view. b. Right carpal bones in anatomical connection (scaphoid, semilunar, and pyramidal). c. Right pisiform, anterior view. d. Right trapezoid, anterior view. $\mathrm{e}$. Right magnum, anterior view. $f$. Right unciform, anterior view. $g$. First lateral phalanx, posterior view; the arrow indicates a possible pathology. $\boldsymbol{h}$. Metacarpals of S. etruscus from La Puebla de Valverde (left) and Crespià (right): h1, Mc II, medial and proximal views; h2, Mc III, anterior and proximal views; h3, Mc IV, medial and proximal views. 

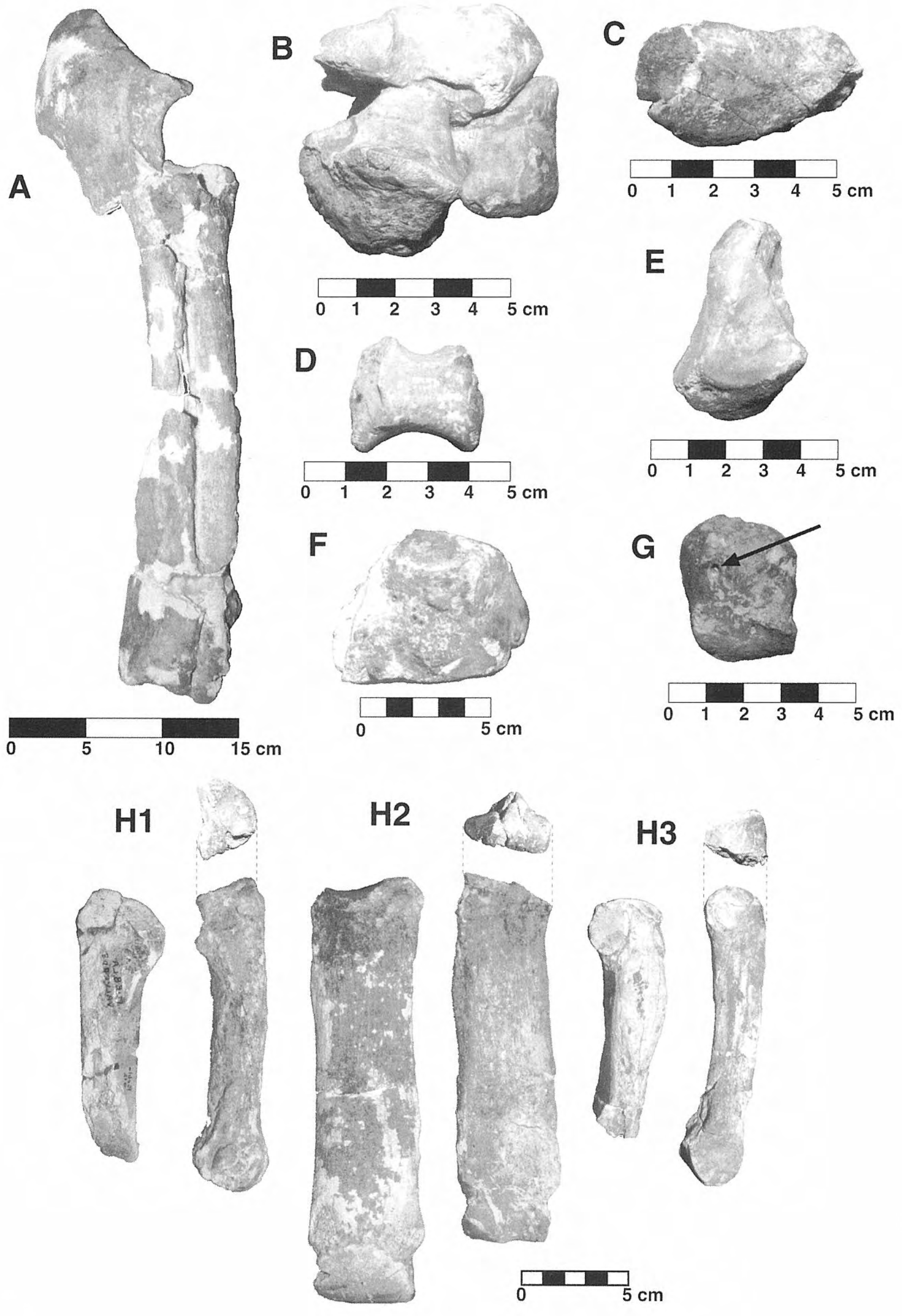


\begin{tabular}{|l|c|c|}
\hline Crespià & & 11,1 \\
\hline Puebla de Valverde (1) & & $12,0-13,0$ \\
\hline \multirow{3}{*}{ Saint Vallier (1) } & Mínima & 13,4 \\
& Media & 12,1 \\
& Máxima & 10,8 \\
\hline & Mínima & 13,2 \\
Senèze (1) & Media & 12,3 \\
& Máxima & 11,4 \\
\hline & Mínima & 13,4 \\
S. etruscus (2) & Media & 12,7 \\
& Máxima & 11,6 \\
\hline & Mínima & 12,6 \\
S. etruscus (3) & Media & 12,3 \\
& Máxima & 12,1 \\
\hline S. hundsheimensis (4) & Mínima & 13,4 \\
& Media & 13,1 \\
& Máxima & 12,8 \\
\hline S. cf hundsheimensis (5) & & $12,53-14,56$ \\
\hline
\end{tabular}

Tabla 6. Índice de gracilidad (DTd x 100 / Longitud) de los radios de $S$. etruscus (1) Guérin y Heintz (1971); (2) a partir de Guérin (1980); (3) a partir de Fortelius et al. (1993); (4) para S. hundsheimensis a partir de Fortelius et al. (1993); (5) para $S$. cf. hundsheimensis a partir de Mazza et al. (1993). Comparative gracility index (DTd $x 100$ / Length) of the radius of $\mathrm{S}$. etruscus from some Spanish, French, Italian, and Dutch localities [after (1) Guérin and Heintz, 1971; (2) Guérin, 1980; (3) Fortelius et al., 1993; (4) for S. hundsheimensis from Fortelius et al., 1993; (5) for S. cf. hundsheimensis from Mazza et al., 1993].

Escafoides (CC-7; Fig. 6b). Está incompleto y permanece fusionado al piramidal y al semilunar. El perfil del borde lateral coincide con los ejemplares de Valdarno (Fortelius et al., 1993, Lám. 5) y Cúllar de Baza (Cerdeño, 1989a, Fig. 3). La faceta para el radio es menos cóncava anteroposteriormente que en el escafoides de La Puebla de Valverde PV (026). El ejemplar de Crespià es más corto (>65 mm) que el PV (026) y no alcanza el valor medio considerado por Fortelius et al., (1993, Tabla 13B) ni el mínimo específico ("D. etruscus etruscus") obtenido por Guérin (1980, Tabla 94). La longitud de la articulación superior $(42,7 \mathrm{~mm})$ coincide con el mínimo de la especie (Guérin, o.c.) y el DAP es ligeramente menor que el de PV (026) (41 mm frente a 43,5 mm).

Semilunar (CC-8; Tabla 8; Fig. 6b). Incompleto. Perímetro anterior coincidente con el de $S$. etruscus (" $D$. etruscus etruscus") de Senèze (Guérin, 1980). Articulación proximal cóncava transversalemnte y aplanada en la Puebla de Valverde. El surco que inferior y posteriormente delimita la faceta lateral superior en los semilunares de La Puebla de Valverde PV (021) y PV (031) y en el CR-9 de El Rincón-1 (Alberdi et al.,1997), no existe en Crespià.

Piramidal (CC-9; Tabla 9; Fig. 6b). La cara anteroexterna es más estrecha y baja que en los ejemplares de La Puebla de Valverde PV (032) y PV (025). En PV (032), la articulación proximal es más aplanada que en CC9. El perímetro distal coincide con el piramidal de Senèze ("D. etruscus etruscus") representado por Guérin (1980, Fig. 64). Las dimensiones son iguales a las mínimas establecidas por Guérin (1980, Tabla 96). Comparativamente, los piramidales de Senèze y Cúllar de Baza (Cerdeño, 1989a, Tabla 5) son mayores que el de Crespià, con menores diferencias en el DAP.

Pisiforme (CC-10; Tabla 10; Fig. 6c). Es comparable al ejemplar de Senèze (Guérin, 1980, Fig 65D-2). El pisiforme de $S$. hundsheimensis de Isernia (Fortelius et al.,1993, Lám 6, Fig. 4) es más asimétrico, robusto y relativamente más largo. El ejemplar de $S$. cf. hundsheimensis de Pietrafitta es, por el contrario, algo más ancho y alto.

Trapezoides (CC-11; Tabla 11; Fig. 6d). Fortelius et al., (1993) observan gran variabilidad en las medidas y proporciones de este hueso en S. etruscus. La faceta para el escafoides es tan cóncava anteroposteriormente como convexa transversalmente. La del magno es aplanada y la del Mc II convexo-cóncava en sentido interno-externo, ovalada y con el vértice desplazado hacia la cara externa.

Magno (CC-12; Fig. 6e). Ejemplar muy incompleto del que sólo se pudo obtener el DT de $38,5 \mathrm{~mm}$. En vista anterior, su perímetro coincide con el de sus homólogos de

\begin{tabular}{|l|l|c|c|c|c|c|c|c|c|c|}
\cline { 3 - 10 } \multicolumn{2}{c|}{} & \multicolumn{3}{c|}{ Olécranon } & \multicolumn{2}{c|}{ Art.prox. } & \multicolumn{2}{c|}{ Diafisis } & \multicolumn{2}{c|}{ Art. distal } \\
\cline { 2 - 11 } \multicolumn{2}{c|}{} & Long. & DT & DAP & DT & DAP & DT & DAP & DT & DAP \\
\hline Crespià & $(440)$ & $(40)$ & $(98)$ & - & 152 & - & $(>28)$ & $(31)$ & - \\
\hline \multicolumn{2}{|l}{ Puebla deValverde (1) } & & & & 78 & & 32 & 43 & 40 & 63 \\
\hline & & & & 77 & & 32 & 38 & - & - \\
\hline \multirow{3}{*}{ St. Vallier } & Min. & & & & 71 & & 29 & 37 & 31 & 50 \\
(1) & Media & & & & 75,7 & & 35,6 & 40 & 36,3 & 58,0 \\
& Max. & & & & 82 & & 36 & 45 & 42 & 66 \\
\hline \multirow{3}{*}{ Senèze (1) } & Min. & & & & 77 & & 33 & 44 & 36 & 60 \\
& Media & & & & 77,7 & & 34,6 & 44,6 & 36,6 & 63,3 \\
& Max. & & & & 79 & & 36 & 45 & 38 & 65 \\
\hline \multirow{3}{*}{ S. etruscus } & Min. & 432 & 37 & 68 & 63 & 112 & 28 & 30 & 26 & 50 \\
(2) & Media & 462,46 & 52,13 & 86,87 & 73,77 & 131,81 & 34,80 & 39,80 & 36,53 & 59,29 \\
& Max. & 501 & 68 & 101 & 82 & 146 & 45 & 52 & 44 & 66 \\
\hline
\end{tabular}

Tabla 7. Dimensiones comparadas de la ulna de S. etruscus de Crespià. (1) Guérin y Heintz (1971); (2) Guérin (1980). Comparative dimensions of the ulna of $\mathrm{S}$. etruscus from Crespià and some Spanish, French, and Italian localities [after (1) Guérin and Heintz, 1971; (2) Guérin, 1980]. 


\begin{tabular}{|c|c|c|c|c|c|}
\hline & DAP & DT & $\mathrm{H}$ & H. articular \\
\hline \multicolumn{2}{|l|}{ Crespià } & 56,0 & - & $(>40)$ & - \\
\hline \multirow{2}{*}{\multicolumn{2}{|c|}{$\begin{array}{l}\text { IPSPV-031 } \\
\text { IPSPV-021 }\end{array}$}} & 63,2 & 44,1 & 48,5 & 48,0 \\
\hline & & 65,2 & 45,1 & 51,1 & 47,1 \\
\hline S. etruscus (1) & $\begin{array}{l}\text { Min. } \\
\text { Media } \\
\text { Max. }\end{array}$ & $\begin{array}{c}56 \\
62,22 \\
68\end{array}$ & $\begin{array}{c}35 \\
44,33 \\
52,5\end{array}$ & $\begin{array}{c}43,5 \\
47,97 \\
51,5\end{array}$ & $\begin{array}{c}51,5 \\
51,75 \\
52\end{array}$ \\
\hline \multicolumn{2}{|c|}{ Cúllar de Baza (2) } & 66 & 48,2 & 45,5 & 47,8 \\
\hline \multicolumn{2}{|c|}{ El Rincón-1 (3) } & 61,6 & 45,6 & 45 & 47,3 \\
\hline \multicolumn{2}{|l|}{ Pietrafitta (4) } & $\begin{array}{c}60 \\
57,2\end{array}$ & $\begin{array}{l}41 \\
48\end{array}$ & $\begin{array}{l}42,7 \\
49,2\end{array}$ & \\
\hline Valdarno (5) & $\begin{array}{l}11 \\
86 \\
\end{array}$ & $\begin{array}{l}70 \\
61 \\
\end{array}$ & $\begin{array}{l}51 \\
45 \\
\end{array}$ & $\begin{array}{l}49 \\
45 \\
\end{array}$ & $\begin{array}{l}50 \\
47 \\
\end{array}$ \\
\hline Olivola (5) & $\begin{array}{l}87 \\
88\end{array}$ & $\begin{array}{l}61 \\
60\end{array}$ & $\begin{array}{l}47 \\
47\end{array}$ & $\begin{array}{l}45 \\
43\end{array}$ & $\begin{array}{l}48 \\
46 \\
\end{array}$ \\
\hline
\end{tabular}

Tabla 8. Dimensiones comparadas del semilunar de $S$. etruscus de Crespià, (1) Guérin (1980); (2) Cerdeño (1989a); (3) Alberdi et al., (1997); (4) Mazza et al., (1993) para S. cf. hudsheimensis; (5) Fortelius et al. (1993).

Comparative dimensions of the semilunate of $\mathrm{S}$. etruscus from Crespià and some Spanish, French and Italian localities [after (1) Guérin, 1980; (2) Cerdeño, 1989a; (3) Alberdi et al., 1997; (4) Mazza et al., 1993, for S. cf. hudsheimensis; (5) Fortelius et al., 1993].

Valdarno (Fortelius et al., 1993, Lám. 7) y Pietrafitta -S. cf. hundsheimensis- (Mazza et al., 1993, Lám 3, Fig. 12).

Unciforme (CC-13; Tabla 12; Fig. 6f). Algo incompleto y de dimensiones ligeramente superiores a las mínimas establecidas, salvo la longitud absoluta que es menor. La cara anterior es trapezoidal, con el borde medial más bajo que el lateral. El perfil inferior es convexo-cóncavo en sentido medial-lateral, distinto al rectilíneo de Senèze (" $D$. etruscus etruscus") figurado por Guérin (1980, Fig. 64G) y al convexo del ejemplar de Valdarno superior (Fortelius et al., 1993, Lám. 7). La faceta para el piramidal es rectangular, con moderada convexidad anteroposterior. La faceta para el magno es trapezoidal, con perfil anteroposterior cóncavoconvexo y transversal aplanado hasta el borde inferior, donde se eleva. La articulación para el Mc IV tiene forma de "L"; la correspondiente al Mc V reducido es oval y pequeña.

Metacarpo (Tablas 13-16). El Mc II izquierdo (CC-14; Fig. 6h1) está completo, aunque deformado; es corto y grácil. Articulación proximal algo cóncava transversalmente. La disposición y morfología de las facetas mediales coinciden con las del fragmento de Cúllar de Baza (Cerdeño, 1989a). La que responde al magno es rectangular, con una altura de $10 \mathrm{~mm}$ y ocupa toda la longitud de la cara. En la mitad anterior, la faceta para el Mc III es trapezoidal; la faceta posterior se reduce a un reborde.

Los Mcs III (CC-15d y CC-15e; Fig. 6h2) están aplastados anteroposteriormente en el tercio distal. Son más robustos que sus homólogos de La Puebla de Valverde (Tabla 14) y presentan unos valores al nivel proximal y de la diáfisis afines a los de un Mc III de Cornillet (Dubar et al., 1978). La faceta para el Mc IV tiene forma de triángulo rectángulo y un DT máximo de 15,6 mm. En Crespià, el acortamiento del metápodo respecto al radio es menor que en la Puebla de Valverde, muy similar al mínimo de Olivola y próximo a los de Senèze y Valdarno. El índice 100 x LMc III / L radio, calculado es el siguiente:

\begin{tabular}{|c|c|c|c|c|c|}
\hline & DT & DAP & Altura & DAP proximal \\
\hline \multicolumn{2}{|l|}{ Crespià } & $(40,9)$ & $(32,8)$ & 42,5 & 27,0 \\
\hline \multicolumn{2}{|l|}{ IPSPV-025 } & 45 & 39,0 & 45,0 & 30,9 \\
\hline \multicolumn{2}{|c|}{ Puebla de Valverde (1) } & $\begin{array}{l}46 \\
-\end{array}$ & $\begin{array}{l}40 \\
-\end{array}$ & $\begin{array}{l}52 \\
51\end{array}$ & \\
\hline St.Vallier (1) & $\begin{array}{l}\text { Mín } \\
\text { Media } \\
\text { Máxima }\end{array}$ & $\begin{array}{c}47 \\
50,0 \\
53 \\
\end{array}$ & $\begin{array}{c}35 \\
35,5 \\
36 \\
\end{array}$ & $\begin{array}{l}- \\
48 \\
- \\
\end{array}$ & \\
\hline Senèze (1) & $\begin{array}{l}\text { Mín. } \\
\text { Media } \\
\text { Máxima }\end{array}$ & $\begin{array}{c}50 \\
50,8 \\
52 \\
\end{array}$ & $\begin{array}{c}33 \\
34,8 \\
38 \\
\end{array}$ & $\begin{array}{c}49 \\
49,8 \\
50 \\
\end{array}$ & \\
\hline \multicolumn{2}{|l|}{ Senèze (2) } & $\begin{array}{c}47,5 \\
45 \\
\end{array}$ & $\begin{array}{c}(35,9) \\
39,2\end{array}$ & $\begin{array}{l}49,2 \\
50,7 \\
\end{array}$ & $\begin{array}{c}33 \\
33,2 \\
\end{array}$ \\
\hline \multicolumn{2}{|c|}{ Cúllar de Baza (2) } & 49,6 & 36,7 & 48 & 31,8 \\
\hline S. etruscus (3) & $\begin{array}{l}\text { Mínima } \\
\text { Media } \\
\text { Máxima }\end{array}$ & $\begin{array}{c}41 \\
49,88 \\
56,5 \\
\end{array}$ & $\begin{array}{l}32 \\
36 \\
40 \\
\end{array}$ & $\begin{array}{c}42 \\
49,19 \\
55\end{array}$ & \\
\hline
\end{tabular}

Tabla 9. Dimensiones comparadas del piramidal de $S$. etruscus de Crespià. (1) Guérin y Heintz (1971); (2) Cerdeño (1989a); (3) Guérin (1980). Comparative dimensions of the pyramidal of $\mathrm{S}$. etruscus from Crespià and some Spanish, French and Italian localities [after (1) Guérin and Heintz, 1971; (2) Cerdeño, 1989a; (3) Guérin, 1980].

- Crespià: 49,42

- La Puebla de Valverde: 47,71-48,64 a partir de Guérin y Heintz (1971, Tabla 4)

- Saint Vallier: 53,62-56,99, a partir de Guérin y Heintz (1971, Tabla 4)

- Senèze: 51,30-60,23, a partir de Guérin y Heintz (1971, Tabla 4)

- Olivola: 50,52-55,64, a partir de Fortelius et al., (1993, Tablas 12a y 21a)

- Valdarno: 51,81-55,71, a partir de Fortelius et al., (1993, Tablas 12a y 21a)

- 53,95, en Guérin (1980, Tabla 117)

- Pietrafitta, S. cf. hundsheimensis: 53,21-54,59, a partir de Mazza et al.,(1993).

- S. hundsheimensis: 51,56, a partir de los valores medios de Fortelius et al., (1993).

El Mc IV (CC-16; Fig. 6h3) es un hueso esbelto, similar métricamente al IGF-116 de Valdarno, salvo por la menor longitud de éste. La epífisis proximal apenas tiene torsión respecto al eje vertical del metápodo, a diferencia de PV

\begin{tabular}{|c|c|c|c|c|}
\hline & Longitud & Anchura & Altura \\
\hline \multicolumn{2}{|l|}{ Crespià } & 56,3 & 22 & (31) \\
\hline S. etruscus (1) & $\begin{array}{l}\text { Mínima } \\
\text { Media } \\
\text { Máxima }\end{array}$ & $\begin{array}{l}58 \\
59 \\
60\end{array}$ & $\begin{array}{c}25 \\
26,10 \\
27\end{array}$ & $\begin{array}{c}35,5 \\
38,20 \\
40,5\end{array}$ \\
\hline \multicolumn{2}{|c|}{ S. hundsheimensis (2) } & 68,8 & 42,5 & 30 \\
\hline \multicolumn{2}{|c|}{ S. cf. hundsheimensis (3) } & $\begin{array}{l}56 \\
57 \\
57\end{array}$ & $\begin{array}{c}41 \\
41,2 \\
36,5\end{array}$ & $\begin{array}{c}24,2 \\
25 \\
28\end{array}$ \\
\hline
\end{tabular}

Tabla 10. Dimensiones comparadas del pisiforme de $S$. etruscus de Crespià. (1) Guérin (1980); (2) Sala y Fortelius (1993); (3) Mazza et al. (1993) para $S$. cf. hundsheimensis.

Comparative dimensions of the pisiform of $\mathrm{S}$. etruscus from Crespià and some Spanish, French and Italian localities [after (1) Guérin, 1980; (2) Sala and Fortelius, 1993; (3) Mazza et al., 1993, for $\mathrm{S}$. $c f$. hundsheimensis]. 


\begin{tabular}{|l|c|c|c|c|}
\cline { 2 - 5 } \multicolumn{1}{c|}{} & Crespià & \multicolumn{3}{c|}{ (Guerin, 1980) } \\
\cline { 2 - 5 } \multicolumn{1}{c|}{} & & Mínima & Media & Máxima \\
\hline Longitud & 32,0 & 35 & 37,94 & 40 \\
\hline Altura & 21,4 & 22 & 27,50 & 32 \\
\hline Anchura & 28,1 & 25,5 & 30,38 & 36 \\
\hline Al / L x 100 (1) & 66,87 & 62,85 & 72,48 & 80 \\
\hline $\mathrm{A} / \mathrm{L} \times 100(1)$ & 87,81 & 72,85 & 80,07 & 90 \\
\hline
\end{tabular}

Tabla 11. Dimensiones del trapezoides de S. etruscus de Crespià comparadas con los datos de Guérin (1980). (1) Índice calculado por Santafé y Casanovas (1989).

Comparative dimensions of the trapezoid of $\mathrm{S}$. etruscus from Crespià and some French and Italian localities [after Guérin, 1980; (1) Index calculated by Santafé and Casanovas, 1989].

(009) donde existe una potente cresta en la cara anterior. La articulación proximal es triangular, más cóncava medial que lateralmente, siendo aplanada en la parte central. En el límite con el lado posterior de la articulación, se observa una superficie de apoyo pequeña y mal delimitada para el Mc V, tal como indica Guérin (1980). En vista medial, las dos facetas para el Mc III están separadas por un estrecho istmo, mientras que en PV (009) están unidas. La sección media de la diáfisis es pentagonal, similar a la del ejemplar de Senèze figurado por Guérin (1980, Fig 68 H-1), pero con una pequeña acanaladura en la cara lateral.

Falanges. La $1^{\text {a }}$ falange del Mc III (CC-17) tiene contorno trapezoidal; su articulación proximal es oval-

\begin{tabular}{|c|c|c|c|c|c|}
\hline & Long. Absoluta & Long. Anatómica & Anchura & Altura \\
\hline \multicolumn{2}{|l|}{ Crespià } & 72,2 & 55,7 & - & - \\
\hline \multicolumn{2}{|c|}{ Puebla de Valverde (1) } & $\begin{array}{l}83 \\
-\end{array}$ & $\begin{array}{l}59 \\
60 \\
\end{array}$ & $\begin{array}{l}62 \\
- \\
\end{array}$ & $\begin{array}{c}46 \\
- \\
\end{array}$ \\
\hline St. Vallier (1) & $\begin{array}{l}\text { Mín } \\
\text { Media } \\
\text { Máxima } \\
\end{array}$ & $\begin{array}{l}82 \\
83 \\
84 \\
\end{array}$ & $\begin{array}{c}59 \\
59,5 \\
60 \\
\end{array}$ & $\begin{array}{c}61 \\
61,5 \\
62 \\
\end{array}$ & 48 \\
\hline Senèze (1) & $\begin{array}{l}\text { Mín } \\
\text { Media } \\
\text { Máxima } \\
\end{array}$ & $\begin{array}{c}79 \\
81,1 \\
83 \\
\end{array}$ & $\begin{array}{c}55 \\
62,9 \\
73 \\
\end{array}$ & $\begin{array}{l}55 \\
59 \\
61 \\
\end{array}$ & $\begin{array}{l}46 \\
47 \\
48 \\
\end{array}$ \\
\hline S. etruscus (2) & \begin{tabular}{|l|} 
Mínima \\
Media \\
Máxima \\
\end{tabular} & $\begin{array}{c}75 \\
81,94 \\
87,5 \\
\end{array}$ & $\begin{array}{c}53 \\
59,50 \\
67 \\
\end{array}$ & $\begin{array}{c}55,5 \\
60,75 \\
73 \\
\end{array}$ & $\begin{array}{c}44 \\
46,72 \\
52 \\
\end{array}$ \\
\hline
\end{tabular}

Tabla 12. Dimensiones comparadas del unciforme de $S$. etruscus de Crespià. (1) Guérin y Heintz (1971); (2) Guérin (1980).

Comparative dimensions of the unciform of $\mathrm{S}$. etruscus from Crespià and some Spanish, French and Italian localities [after (1) Guérin and Heintz, 1971; (2) Guérin, 1980].

elíptica y con un ligero entrante en el centro del borde posterior. La $2^{a}$ falange (CC-18) es de contorno más rectangular; la articulación distal presenta un perfil convexo, asimétrico en sentido dorsopalmar.

La $3^{\text {a }}$ falange central (CC-19) tiene la faceta proximal alargada, aplanada y con DAP reducido. El borde distal presenta abundantes forámenes nutricios.

La $1^{\text {a }}$ falange del Mc IV (CC-20; Fig. 6g) tiene en el cuadrante superoexterno posterior un orificio poroso internamente, con el área que lo circunda algo albultada. Esta condición nos recuerda las observadas por Miralles y

\begin{tabular}{|c|c|c|c|c|c|c|c|c|c|c|}
\hline & & \multicolumn{2}{|c|}{ Epífisis proximal } & \multicolumn{2}{|c|}{ Art. proximal } & \multicolumn{2}{|c|}{ Diafisis } & \multicolumn{2}{|c|}{ Art. distal } \\
\hline & & Long. & DT & DAP & DT & DAP & DT & DAP & DT & DAP \\
\hline \multicolumn{2}{|l|}{ Crespià } & 154 & (30) & $(35)$ & (24) & $(32)$ & (23) & $(28)$ & (33) & 39 \\
\hline \multicolumn{2}{|c|}{ Puebla deValverde (1) } & & $\begin{array}{l}47 \\
45\end{array}$ & $\begin{array}{l}39 \\
39 \\
\end{array}$ & & & & & & \\
\hline $\begin{array}{l}\text { St. } \\
\text { (1) }\end{array}$ & \begin{tabular}{|l|} 
Mín \\
Media \\
Máxima \\
\end{tabular} & & $\begin{array}{c}33 \\
38,2 \\
43 \\
\end{array}$ & $\begin{array}{c}40 \\
42,7 \\
46 \\
\end{array}$ & & & & & & \\
\hline Senèze (1) & \begin{tabular}{|l|} 
Mín \\
Media \\
Máxima \\
\end{tabular} & & $\begin{array}{c}34 \\
39,7 \\
43 \\
\end{array}$ & $\begin{array}{c}35 \\
41,3 \\
46 \\
\end{array}$ & & & & & & \\
\hline \multicolumn{2}{|l|}{ Senèze (2) } & $\begin{array}{l}179 \\
180 \\
181\end{array}$ & $\begin{array}{c}34,6 \\
34,7 \\
30\end{array}$ & $\begin{array}{c}47,7 \\
48 \\
40,6\end{array}$ & $\begin{array}{c}25 \\
26,5 \\
24\end{array}$ & $\begin{array}{l}41,3 \\
40,2 \\
34,8\end{array}$ & $\begin{array}{l}34 \\
36 \\
32\end{array}$ & $\begin{array}{l}21 \\
21 \\
20\end{array}$ & $\begin{array}{c}38,4 \\
37 \\
34,8\end{array}$ & $\begin{array}{c}38 \\
38 \\
36,4\end{array}$ \\
\hline \multicolumn{2}{|c|}{ Cullar Baza (2) } & - & 34,9 & 48,1 & 24,4 & 42,8 & 32,7 & & & \\
\hline Solihac (2) & & 192,4 & 33,2 & 47,3 & 22,9 & (39) & 34,2 & 20 & 40,7 & $(38,5)$ \\
\hline \multicolumn{2}{|c|}{ Venta Micena (3) } & & 39,2 & 36 & & & 28 & 19 & & (35) \\
\hline $\begin{array}{l}\text { S. etruscus } \\
\text { (4) }\end{array}$ & $\begin{array}{l}\text { Mínima } \\
\text { Media } \\
\text { Máxima } \\
\end{array}$ & $\begin{array}{l}162 \\
176 \\
186 \\
\end{array}$ & $\begin{array}{c}27 \\
37,34 \\
47 \\
\end{array}$ & $\begin{array}{c}34 \\
40,66 \\
46,5 \\
\end{array}$ & & & $\begin{array}{c}24 \\
32,47 \\
38 \\
\end{array}$ & $\begin{array}{c}17 \\
22,61 \\
28 \\
\end{array}$ & $\begin{array}{c}30 \\
38,18 \\
45,5\end{array}$ & $\begin{array}{c}36 \\
37,89 \\
41\end{array}$ \\
\hline \multicolumn{2}{|l|}{ Capitone (5) } & 172 & & 40 & & & & & 36 & 37 \\
\hline Valdarno (6) & $\begin{array}{l}11 \\
102 \\
103\end{array}$ & $\begin{array}{c}166 \\
- \\
173\end{array}$ & $\begin{array}{l}44 \\
46 \\
43\end{array}$ & $\begin{array}{l}32 \\
40 \\
39\end{array}$ & $\begin{array}{l}39 \\
- \\
39\end{array}$ & $\begin{array}{l}32,4 \\
39,3 \\
37,8\end{array}$ & $\begin{array}{l}31 \\
-33\end{array}$ & $\begin{array}{l}19 \\
- \\
37\end{array}$ & $\begin{array}{l}31 \\
-33\end{array}$ & \\
\hline Olivola (6) & 104 & - & 41 & 37 & (37) & 33,5 & $(36)$ & $(15)$ & - & \\
\hline
\end{tabular}

Tabla 13. Dimensiones comparadas del Mc II de S. etruscus de Crespià. (1) Guérin y Heintz (1971); (2) Cerdeño (1989a); (3) Santafé y Casanovas (1987); (4) Guérin (1980); (5) Ambrosetti (1972); (6) Fortelius et al. (1993).

Comparative dimensions of the Mc II of S. etruscus from Crespià and some Spanish, French and Italian localities [after (1) Guérin and Heintz, 1971; (2) Cerdeño, 1989a; (3) Santafé and Casanovas, 1987; (4) Guérin, 1980; (5) Ambrosetti, 1972; (6) Fortelius et al., 1993]. 


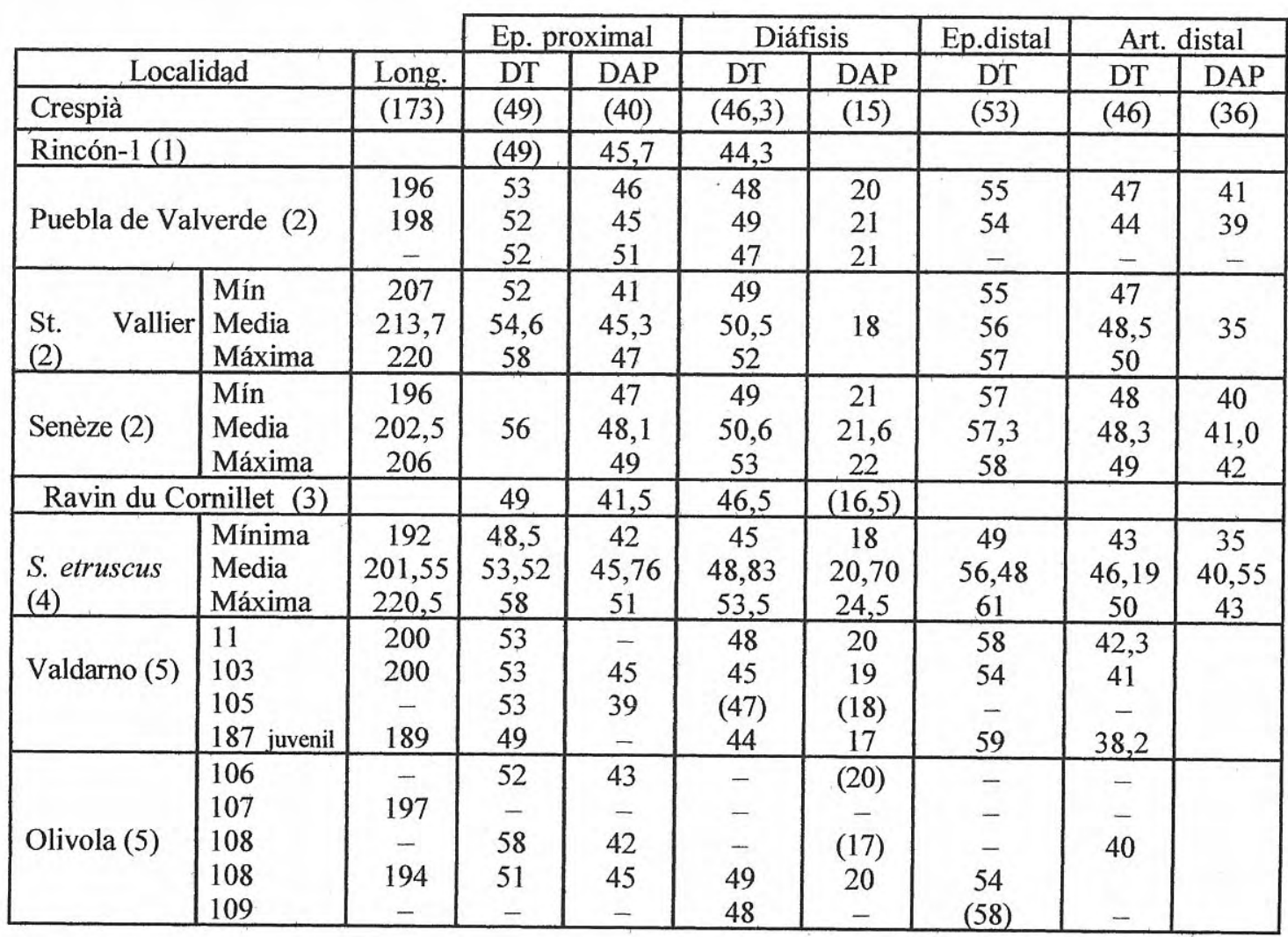

Tabla 14. Dimensiones comparadas del Mc III de S. etruscus de Crespià. (1) Alberdi et al. (1997); (2) Guérin y Heintz (1971); (3) Dubar et al. (1978); (4) Guérin (1980); (5) Fortelius et al. (1993).

Comparative dimensions of the Mc III of S. etruscus from Crespià and some Spanish, French and Italian localities [after (1) Alberdi et al., 1997; (2) Guérin and Heintz, 1971; (3) Dubar et al., 1978; (4) Guérin, 1980; (5) Fortelius et al., 1993].

Crusafont (1952, Lám. XV) en dos falanges de Gazellospira de Villarroya, atribuidas a un proceso de osteomielitis.

Cintura pelviana. Sólo se conserva el coxal derecho (CC-21). Le falta buena parte del isquion y presenta deteriorado el borde de la cavidad acetabular $(80 \mathrm{~mm} \times(60)$ $\mathrm{mm}$ ). El ilion es pequeño y triangular. La tuberosidad sacral es más gruesa y rugosa que la coxal, con una distancia entre los puntos más distantes de $140 \mathrm{~mm}$. La altura desde la cresta ilíaca al borde opuesto es de $61 \mathrm{~mm}$.

Extremidades posteriores. Desaparecieron por la acción de las máquinas excavadoras de la cantera, de forma que sólo se preservó la cabeza de articulación proximal del fémur derecho (CC-20), cuyos diámetros máximo y mínimo son $72 \mathrm{~mm}$ y $51 \mathrm{~mm}$, respectivamente.

Como resumen de esta descripción comparativa, se puede concluir que los restos de $S$. etruscus de Crespià son comparables a los ya conocidos de la especie, presentando algunas particularidades morfológicas atribuibles a variación intraespecífica. En particular, presentan mayor afinidad con los restos villafranquienses de España, manteniendo una talla relativamente pequeña con respecto a la media europea, tal como ya había sido notado para otros restos españoles (Cerdeño, 1993).

Con respecto a $S$. hundsheimensis, especie estrechamente relacionada con $S$. etruscus (Fortelius et al., 1993; Mazza et al., 1993), la morfología craneal comparada ratifica las afinidades en lo referente a la disposición de las apófisis óticas y al perímetro trapezoidal de la cara occipital (Fortelius et al., o.c.) y las diferencias por éstos indicadas relativas al mayor desarrollo de los nasales, a la mejor señalización de las inserciones para los cuernos y al acortamiento de la porción facial en $S$. hundsheimensis.

Respecto a la dentición superior, $S$. hundsheimensis de Isernia presenta premolares con pliegues secundarios más desarrollados y complejos (Sala y Fortelius, 1993, Lám. 2). A nivel postcraneal, el ejemplar de S. etruscus de Crespià ostenta un nivel de acortamiento del radio respecto al Mc III ligeramente superior y se distingue por la mayor gracilidad del primero, por las reducidas dimensiones y proporciones de los huesos carpales comparados, por la menor longitud de los metacarpos, por la mayor gracilidad de los laterales y mayor robustez de los centrales (Tabla 15). Respecto a $S$. cf. hundsheimensis de Pietrafitta, Pirro y Westerhoven (Mazza et al.,1993), el ejemplar de S. etruscus de Crespià comparte la morfometría de la escápula y la longitud de los metacarpos laterales y se distingue, además de por las diferencias indicadas para S. hundsheimensis, por el mayor grado de acortamiento de sus radios respecto al Mc III.

El esqueleto hallado en Crespià perteneció a un animal adulto de $S$. etruscus, de talla pequeña-media y masa corporal estimada en $1200 \mathrm{~kg}$ (a partir de la fórmula 


\begin{tabular}{|c|c|c|c|c|c|c|c|c|c|}
\hline & & \multicolumn{2}{|c|}{ Ep. prox. } & \multicolumn{2}{|c|}{ Diafisis } & Ep.distal & \multicolumn{2}{|c|}{ Art.distal } \\
\hline & & Longitud & DT & DAP & DT & DAP & DT & DT & DAP \\
\hline \multicolumn{2}{|l|}{ Crespià } & 146,6 & 38 & 32,4 & 23,2 & 20 & (35) & (30) & (27) \\
\hline \multicolumn{2}{|l|}{ IPSPV-009 } & - & 41 & 34,2 & 32,5 & 20,0 & & & \\
\hline \multicolumn{2}{|c|}{ Puebla de Valverde (1) } & $\begin{array}{c}162 \\
-\end{array}$ & $\begin{array}{l}42 \\
44\end{array}$ & $\begin{array}{l}32 \\
35\end{array}$ & $\begin{array}{l}30 \\
-\end{array}$ & 22 & $\begin{array}{l}38 \\
-\end{array}$ & $\begin{array}{l}35 \\
-\end{array}$ & $\begin{array}{l}36 \\
-\end{array}$ \\
\hline St. Vallier (1) & $\begin{array}{l}\text { Mín } \\
\text { Media } \\
\text { Máxima }\end{array}$ & 176 & $\begin{array}{c}41 \\
42,8 \\
45\end{array}$ & $\begin{array}{c}36 \\
37,7 \\
40\end{array}$ & $\begin{array}{c}30 \\
30,5 \\
31\end{array}$ & 21 & 46 & 42 & - \\
\hline Senèze (1) & $\begin{array}{l}\text { Mín. } \\
\text { Media } \\
\text { Máxima }\end{array}$ & $\begin{array}{l}158 \\
167 \\
171 \\
\end{array}$ & $\begin{array}{c}40 \\
41,3 \\
45 \\
\end{array}$ & $\begin{array}{c}33 \\
35,8 \\
38 \\
\end{array}$ & $\begin{array}{c}28 \\
31,1 \\
34\end{array}$ & $\begin{array}{c}20 \\
22,0 \\
24\end{array}$ & $\begin{array}{c}40 \\
42,7 \\
46\end{array}$ & $\begin{array}{c}34 \\
37,0 \\
39\end{array}$ & $\begin{array}{c}37 \\
37,9 \\
39\end{array}$ \\
\hline \multicolumn{2}{|l|}{ Capitone (2) } & & - & 43 & & & & 38 & 38 \\
\hline $\begin{array}{l}\text { S. etruscus } \\
\text { (3) }\end{array}$ & $\begin{array}{l}\text { Mínima } \\
\text { Media } \\
\text { Máxima }\end{array}$ & $\begin{array}{c}157 \\
166,26 \\
177\end{array}$ & $\begin{array}{c}40 \\
41,98 \\
45,5\end{array}$ & $\begin{array}{c}32 \\
36,25 \\
40\end{array}$ & $\begin{array}{c}27 \\
31,95 \\
38\end{array}$ & $\begin{array}{c}19 \\
22 \\
26,5\end{array}$ & $\begin{array}{c}38 \\
42,17 \\
46\end{array}$ & $\begin{array}{c}34 \\
37,72 \\
42\end{array}$ & $\begin{array}{c}31,5 \\
37,56 \\
43\end{array}$ \\
\hline Valdarno (4) & $\begin{array}{l}11 \\
110 \\
\end{array}$ & $\begin{array}{l}165 \\
169 \\
\end{array}$ & $\begin{array}{l}37 \\
43 \\
\end{array}$ & $\begin{array}{l}37 \\
38 \\
\end{array}$ & $\begin{array}{l}32 \\
39 \\
\end{array}$ & $\begin{array}{l}17 \\
20 \\
\end{array}$ & $\begin{array}{l}42 \\
46 \\
\end{array}$ & $\begin{array}{c}33 \\
(35) \\
\end{array}$ & \\
\hline Olivola (4) & $\begin{array}{l}111 \\
112 \\
113 \\
114 \\
115 \\
116 \\
117\end{array}$ & $\begin{array}{c}176 \\
157 \\
- \\
- \\
- \\
(149) \\
-\end{array}$ & $\begin{array}{c}40 \\
(37) \\
- \\
- \\
(36) \\
(38) \\
39\end{array}$ & $\begin{array}{c}41 \\
40 \\
- \\
- \\
(29) \\
32 \\
(31)\end{array}$ & $\begin{array}{c}36 \\
35 \\
34 \\
(39) \\
(37) \\
32 \\
(36)\end{array}$ & $\begin{array}{c}19 \\
17 \\
19 \\
- \\
(12) \\
21 \\
(17)\end{array}$ & $\begin{array}{c}42 \\
40 \\
37 \\
- \\
- \\
33 \\
-\end{array}$ & $\begin{array}{c}36 \\
32 \\
34 \\
- \\
- \\
(32) \\
-\end{array}$ & \\
\hline \multicolumn{2}{|l|}{ Isernia (5) } & $\begin{array}{c}167 \\
- \\
-\end{array}$ & $\begin{array}{l}45,5 \\
41,8 \\
42,0\end{array}$ & $\begin{array}{l}44,5 \\
44,3 \\
44,0\end{array}$ & $\begin{array}{c}33,7 \\
- \\
-\end{array}$ & $\begin{array}{l}22 \\
- \\
-\end{array}$ & $\begin{array}{c}42,2 \\
- \\
-\end{array}$ & $\begin{array}{l}37 \\
- \\
-\end{array}$ & \\
\hline
\end{tabular}

Tabla 15. Dimensiones comparadas del McIV de S. etruscus de Crespià. (1) Guérin y Heintz (1971); (2) Ambrosetti (1972); (3) Guérin (1980); (4) Fortelius et al. (1993); (5) Sala y Fortelius (1993) para S. hundsheimensis. Comparative dimensions of the Mc IV of S. etruscus from Crespià and some Spanish, French and Italian localities [after (1) Guérin and Heintz, 1971; (2) Ambrosetti, 1972; (3) Guérin, 1980; (4) Fortelius et al., 1993; (5) Sala and Fortelius, 1993, for S. hundsheimensis].

de Legendre, 1986), inferior a la calculada por Cerdeño (1998, Fig. 7) a partir de la media europea, estimada en $1763,9 \mathrm{~kg}$ (datos de E.C.).

Las proporciones de los huesos largos son gráciles, aunque los metápodos muestran unas epífisis relativamente anchas.

Esta característica se ha observado en otras especies de rinocerontes y se ha interpretado como una mayor adaptación a suelos blandos (Cerdeño, 1989b, 1998), lo cual estaría de acuerdo con las inferencias paleoambientales deducidas para Crespià (Villalta y Vicente, 1972; Roiron, 1983; Galobart, 1996). Según dichos autores, el hábitat de Crespià estaría constituido por un bosque húmedo y abierto donde, salvo algunas extensiones herbáceas pobladas por gramíneas -Arundo sp. (Villalta y Vicente, o.c.)-, predominaba una vegetación propia de las riberas de ríos y lagos, con Populus alba, Alnus glutinosa o Fraxinus excelsior. El nivel de humedad ambiental era alto y el régimen de temperaturas permitía la asociación de especies propias de un clima fresco de tipo atlántico -Ilex aquifolium o Quercus cerris- con otras de tipo mediterráneo -Quercus ilex- y algunas propiamente termófilas, como Laurus canariensis (Villalta y Vicente, o.c.).

\begin{tabular}{|c|c|c|c|c|}
\hline \multirow{2}{*}{\multicolumn{2}{|c|}{ Crespià }} & $\mathrm{Mc}$ II & Mc III & Mc IV \\
\hline & & $(14,93)$ & $(26,76)$ & 15,82 \\
\hline \multicolumn{2}{|c|}{ Puebla de Valverde (1) } & & $\begin{array}{l}24,48 \\
24,74 \\
\end{array}$ & 18,51 \\
\hline \multicolumn{2}{|l|}{ Saint Vallier (1) } & & $\begin{array}{l}23,67 \\
23,63 \\
23,63 \\
\end{array}$ & $\begin{array}{l}17,04 \\
17,32 \\
17,61 \\
\end{array}$ \\
\hline \multicolumn{2}{|l|}{ Senèze (1) } & & $\begin{array}{c}25 \\
24,98 \\
25,72 \\
\end{array}$ & $\begin{array}{l}17,72 \\
18,62 \\
19,88 \\
\end{array}$ \\
\hline S. etruscus (2) & $\begin{array}{l}\text { Máxima } \\
\text { Media } \\
\text { Mínima }\end{array}$ & $\begin{array}{l}14,81 \\
18,44 \\
20,43\end{array}$ & $\begin{array}{l}23,43 \\
24,22 \\
24,26\end{array}$ & $\begin{array}{l}17,19 \\
19,21 \\
21,46\end{array}$ \\
\hline S. etruscus (3) & Media & 19,66 & 23,96 & 21,78 \\
\hline S. hundsheimensis & Media & $(19,59)$ & 24,29 & 21,08 \\
\hline \multicolumn{2}{|c|}{ S. cf. hundsheimensis (4) } & $19,18-19,43$ & $23,11-23,95$ & $18,50-19,33$ \\
\hline
\end{tabular}

Tabla 16. Índice de gracilidad (DTd x 100 / Longitud) de los metacarpos de $S$. etruscus, $S$. hundsheimensis y $S$. cf. hundsheimensis a partir de: (1) Guérin y Heintz (1971: Tabla 4); (2) Guérin (1980); (3) Fortelius et al. (1993); (4) Mazza et al. (1993).

Comparative gracility index (DTd $x 100 /$ Length) of the metacarpals of $\mathrm{S}$. etruscus, S. hundsheimensis and S. cf. hundsheimensis from some Spanish, French, Italian and Dutch localities [after (1) Guérin and Heintz, 1971; (2) Guérin, 1980; (3) Fortelius et al., 1993; (4) Mazza et al., 1993]. 


\section{CONCLUSIONES}

Este estudio confirma la atribución preliminar del rinoceronte de Crespià a la especie Stephanorhinus etruscus, realizada por uno de los firmantes (Galobart, 1996), y permite aumentar el conocimiento de la especie, escasamente representada en España.

El estudio morfométrico detallado de los restos permite resaltar las siguientes conclusiones:

- En conjunto, presentan una morfología comparable a la de otros restos de $S$. etruscus y contribuyen al conocimiento de la variabilidad de esta especie. Las dimensiones son, en general, inferiores a la media europea establecida para la especie.

- La morfología craneal es característica de S. etruscus, de acuerdo con las descripciones de Guérin (1980), Mazza (1988) y Fortelius et al. (1993). A nivel métrico, sólo dos valores superan los máximos específicos y uno es inferior a éstos, el resto no alcanza la media.

- La mandíbula presenta la sínfisis acortada y las ramas ascendentes relativamente más bajas con respecto a otros restos villafranquienses, como los de Las Higueruelas y Valdarno.

- Dentición superior de talla pequeña, respecto a la media general y a los otros restos españoles; los dientes inferiores son pequeños respecto a la media, salvo el p2, que es más ancho que los de otras localidades españolas.

- La serie premolar inferior es relativamente larga en relación con los valores medios de la especie.

- Morfológicamente, los P4 y M1 de Crespià tienen pliegue del metacono y falta el del mesostilo en los segundos. En la superficie oclusal, los ganchos son simples y las cristas, débiles. En los dientes del Pleistoceno inferior peninsular los pliegues secundarios están más desarrollados, tal y como expone Cerdeño (1993).

- El perfil de los valles en el m3 es distinto al mayoritario en "U" ancha considerado por Fortelius et al., (1993, Tabla 4).

- En el m3, no hay diferencia de nivel entre los valles, al contrario de lo que ocurre en sendos ejemplares de Venta Micena (Santafé y Casanovas, 1987) y Cúllar de Baza (Cerdeño, 1989a).

- El radio y la ulna son más cortos y gráciles que los de La Puebla de Valverde aunque muy similares morfológicamente (Guérin y Heintz, 1971; Santafé y Casanovas, 1989).

- Los huesos carpales apenas superan en talla, en algunos casos, los mínimos específicos establecidos. A nivel morfológico, sólo la cara lateral del semilunar no concuerda con las características de S. etruscus (Guérin, 1980; Mazza, 1988; Cerdeño, 1989a; Fortelius et al., 1993; Alberdi et al., 1997).

- Los metacarpos laterales de Crespià presentan, respecto a los de La Puebla de Valverde, diáfisis más esbeltas, menos curvadas e impresiones musculares más débiles.

- En general, los metápodos son gráciles pero con las epífisis relativamente anchas, ya que se advierte menor diferencia de los DTs de las epífisis que de la longitud respecto a los mínimos de la especie. Esta particularidad podría interpretarse como una mayor adaptación a un suelo blando, tal como se ha inferido para otras especies (Cerdeño, 1989b, 1998), lo cual estaría en concordancia con la reconstrucción de un hábitat lacustre con un alto grado de humedad para Crespià (Villalta y Vicente, 1972; Roiron, 1983; Galobart, 1996).

\section{AGRADECIMIENTOS}

Los autores desean expresar su agradecimiento a los Drs. José Vicente Santafé, M $^{\mathrm{a}}$. Lourdes Casanovas y Jordi Agustí, del Institut de Paleontologia M. Crusafont de Sabadell, por sus valiosos comentarios sobre el manuscrito. A la Dra. Begoña Sánchez, del Museo Nacional de Ciencias Naturales, por haber facilitado parte de la bibliografía consultada. Trabajo financiado por el proyecto de la DGICYT PB97-0157 "Cambio climático y sucesiones faunísticas en el Plio-pleistoceno del Levante español" del Ministerio de Educación y Cultura.

\section{BIBLIOGRAFÍA}

Alberdi, M.T., Cerdeño, E., López Martínez, N., Morales, J. y Soria, M.D. 1997. La fauna villafranquiense del Rincón-1. Estudios Geológicos, 53, 1-93.

Ambrosetti, P. 1972. Lo scheletro di Dicerorhinus etruscus (Falconer) di Capitone (Umbria meridionale). Geológica romana, 11, 177-198.

Boeuf, O. 1994. Le Dicerorhinus etruscus (Rhinocerotidae, Mammalia) du site Pliocène-Supérieur de Chilhac (Haute-Loire, France). Geobios, 28, 383-391.

Bouchud, J. 1966. Les Rhinocéros. Faunes et Flores préhistoriques. Atlas de Préhistoire. Tome III, Editions N. Boubée et Cie., Paris, 174-193.

Cerdeño, E. 1989a. Rhinocerotidae (Mammalia, Perissodactyla) de la cuenca de Guadix-Baza. Trabajos sobre el Neógeno-Cuaternario, 11, 273-286.

Cerdeño, E. 1989b. Revisión de la sistemática de los rinocerontes del Neógeno de España. Editorial de la Universidad Complutense de Madrid. Colección Tesis Doctorales, 306/89, 1-429.

Cerdeño, E. 1993. Remarks on the Spanish Plio-Pleistocene Stephanorhinus etruscus (Rhinocerotidae). Comptes Rendus de l'Académie de Sciences de Paris, 317, 13631367.

Cerdeño, E. 1998. Diversity and evolutionary trends in the Family Rhinocerotidae (Perissodactyla). Palaeogeography, Palaeoclimatology, Palaeoecology, 141, 1334.

Dubar, M., Guérin, C. et Heintz, E. 1978. Les nouveaux gisements Villafranchiens du ravin de Cornillet (Moustiers, Sainte Marie, Alpes de Haute Provence, France) et leur contexte géologique. Geobios, 11, 367381.

Falconer, H. 1868. On the European Pliocene and Postpliocene species of the genus Rhinoceros. Paleontological Memoirs and Notes (Ed. C. Murchinson), 2, 309-403. 
Fleta, J., Grellet, B., Philip, H., Escuer, J., Goula, X. i Bousquet, J.C. 1996. Les deformacions tectòquines en els materials plioquaternaris de la depressió de TortellàBesalú. Quaderns del Centre d'Estudis Comarcals de Banyoles, 17. Geologia de la conca lacustre de BanyolesBesalú. Enquadrament, materials i evolució, 99-112.

Fortelius, M., Mazza, P. and Sala, B. 1993. Stephanorhinus (Mammalia: Rhinocerotidae) of the western European Pleistocene, with a revision of $S$. etruscus (Falconer, 1868). Palaeontographia Italica, 80, 63-155.

Galobart, A. 1996. Estudi de la fauna de mamífers dels jaciments del Plistocè inferior d'Incarcal (Crespià, Pla de l'Estany): sistemàtica, tafonomia i paleoecologia. Tesis doctoral inédita. Universitat Autònoma de Barcelona, 1-375.

Galobart, A., Maroto, J., Menéndez, E., Ros, X., Gaete, R. y Colomer, F. 1990. El yacimiento del Pleistoceno Inferior de Incarcal (Crespià, Girona). Comunicaciones de la Reunión de Tafonomía y Fosilización, Ed. U.C.M. y C.S.I.C., Madrid, 161-167.

Geurts, M. A. 1977. Premières données à l'étude palynologique des dépôts calcareux quaternaires de Catalogne. Acta Geológica Hispánica, 12 (4-6), 86-89.

Geurts, M. A. 1979. Approche palynostratigraphique des dépôts calcareux dans la region de Banyoles-Besalú (Catalogne). Actas de la IV Reunión del Grupo de Trabajo del Cuaternario, 107-115.

Guérin, C. 1972. Une nouvelle espèce de rhinocéros (Mammalia, Perissodactyla) à Vialette (Haute-Loire, France) et dans d'autres gisements du Villafranchien inférieure européen: Dicerorhinus jeanvireti n. sp. Documents des Laboratoires de Geólogie de la Faculté des Sciences de Lyon, 49, 1-156.

Guérin, C. 1980. Les rhinocéros (Mammalia-Perissodactyla) du Miocene terminal au Pleistocène supérieur en Europe Occidentale. Comparaison avec les espèces actuelles. Documents des Laboratoires de Géologie de la Faculté des Sciences de Lyon, 79, 1-1185.

Guérin, C. et Heintz, E. 1971. Dicerorhinus etruscus (Falconer, 1859), Rhinocerotidae, Mammalia, du Villafranchien de La Puebla de Valverde (Teruel, Espagne). Bulletin du Muséum National d'Histoire Naturelle, 18, 13-23.

Julià, R. 1980. La conca lacustre de Banyoles-Besalú. Monografies del Centre d'Estudis Comarcals de Banyoles. Banyoles, 1-187.

Julià, R. y Villalta, J.F. 1984. El yacimiento del Pleistoceno inferior de Crespià (Girona, NE de la Penínsla Ibérica). Acta Geológica Hispánica, 19 (2), 129-138.

Kretzoi, M. 1942. Bemerkungen zum System der nachmiozänen Nashorn-Gattungen. Földtani Közloni, 72, 309-318.

Legendre, S. 1986. Analysis of mammalian communities from the late Eocene and Oligocene of Southern France. Palaeovertebrata, 16, 191-212.

Maroto, J. i Galobart A. 1992. Incarcal (Crespià, Pla de l'Estany): un jaciment representatiu de la fauna de grans mamífers del Pleistocè inferior. Tribuna d'Arqueologia, 1990-1991, 7-16.

Mazo, A. 1995. Stephanorhinus etruscus (Perissodactyla,
Mammalia) en el Villafranquiense inferior de Las Higueruelas, Alcolea de Calatrava (Ciudad Real). Estudios Geológicos, 51, 285-290.

Mazo, A. y Torres, T. 1990. El pozo de Piedrabuena, un nuevo yacimiento de vertebrados pliocenos en el Campo de Calatrava (Ciudad Real). Paleontologia i Evolució, 23, 213-222.

Mazo, A., Sesé, C., Ruiz Bustos, A. y Peña, J.A. 1985. Geología y Paleontología de los yacimientos pliopleistocenos de Huéscar (Depresión de Guadix-Baza, Granada). Estudios Geológicos, 41, 467-493.

Mazza, P. 1988. The Tuscan early pleistocene rhinoceros Dicerorhinus etruscus. Palaeontographia Italica, 75,187.

Mazza, P., Sala, B. and Fortelius, M. 1993. A small latest Villafranchian (late Early Pleistocene) rhinoceros from Pietrafitta (Perugia, Umbria, Central Italy), with notes on the Pirro and Westerhoven rhinoceroses. Palaeontographia italica, 80, 25-50.

Miralles, A. y Crusafont, M. 1952. Algunos casos de paleopatología y de paleoteratología en artiodáctilos del Terciario español. Estudios Geológicos, 16, 225-231.

Roiron , P. 1983. Nouvelle étude de la macroflore PlioPléistocène de Crespià (Catalogne, Espagne). Geobios, 16, 687-715.

Sala, B. and Fortelius, M. 1993. The rhinoceroses of Isernia La Pineta (early Middle Pleistocene, Southern Italy). Paleontographia Italica, 8, 157-174.

Santafé, J.V. y Casanovas, Mª.L. 1987. Dicerorhinus etruscus brachycephalus (Mammalia, Perissodactyla) de los yacimientos pleistocénicos de la cuenca Guadix-Baza (Venta Micena y Huéscar) (Granada, España). Paleontologia $i$ Evolució, Memoria especial, 1, 237. 254.

Santafé, J.V. y Casanovas, $\mathrm{M}^{\mathrm{a}}$.L. 1989. Dicerorhinus hemitoechus (Falconer, 1968) (Mammalia, Perissodactyla) del yacimiento Pleistocénico de la Cova del Gegant (Garraf, Barcelona). Empúries, 48-50, 310-322.

Schroeder, H. 1903. Die Wirbelthier-Fauna des Mosbacher Sandes.1. Gattung Rhinoceros. Abhandlungen der Koniglich Preussischen Geolgischen Landesanstal., 18, 1-143.

Suc, J.P. 1982. Contribution a la connaissance du Pliocène et du Pléistocène inférieur des regions méditerranéennes d'Europe Occidentale par l'analyse palinologique des dépôts du Langedoc-Roussillon (Sud de France) et la Catalogne (Nord-Est de l'Espagne). Tesis Doctoral. Université des Sciences et Techniques du Languedoc. 2 vol., 40 figs., 13 Tablas, 1-198.

Villalta, J.F. 1952. Contribución al conocimiento de la fauna de mamíferos fósiles del Plioceno de Villarroya (Logroño). Boletín del Instituto Geológico y Minero de España, 64, 1-201.

Villalta, J.F.y Vicente, J. 1972. Una flora del Cuaternario antiguo en las cercanías de Crespià. Acta Geológica Hispánica, 7 (4), 120-128.

Manuscrito recibido: 27 de junio, 2000 Manuscrito aceptado: 6 de noviembre, 2000 